\title{
Synthesis of new phosphonoglycine backbone units for the development of phosphono nucleic acids.
}

\author{
Bogdan Doboszewski, ${ }^{[a, b]}$ Elisabetta Groaz, ${ }^{[b]}$ and Piet Herdewijn*[b]
}

Keywords: Nucleotides / Phosphonates / Phosphono peptide nucleic acid (pPNA)

A series of phosphono-modified backbone mimics based on achiral and chiral $N$-(dihydroxypropyl)glycine units were obtained by sequential addition of phosphonate and nucleobase moieties to suitably protected dihydroxypropylamines. Simple synthetic strategies enabled the preparation of various target derivatives that will be useful as building blocks for the preparation of new synthetic polymers containing a phosphonate internucleotide linkage in place of the standard phosphodiester bond.

\section{Introduction}

Genetic information in all living organisms is coded in sequences of pyrimidines and purines connected to a backbone of phosphorylated 2-deoxy-(D)-ribofuranoses (in DNA) or $(D)$ ribofuranoses (in RNA). The development of unnatural nucleic acids, especially comprising sugar mimics in their backbone replacing the natural furanosyl units, for the formation of new oligomeric pairing systems represents an important avenue of research in synthetic biology and in the search for the origin of life. ${ }^{[1]}$ Studies aiming at defining an etiology of nucleic acids have demonstrated that canonical Watson-Crick base pairing can be supported by chemically simplified backbone structures. ${ }^{[2-4]}$ Among such modifications, one relevant class of DNA mimics consists of Peptide Nucleic Acids (PNAs), ${ }^{[5]}$ linear pseudo-peptide backbones connected to nucleobases through methylene carbonyl linkages. Many structural variants, closely related to N-(2aminoethyl)-glycine PNA, ${ }^{[6]}$ were synthetized and found to be capable of stable antiparallel homoduplex and triple helix formation (Figure 1). ${ }^{[5]}$ PNA strongly hybridizes with natural complements in a sequence-specific manner, forming PNA.DNA and PNA.RNA binary complexes with increased specificity and thermal stability compared to unmodified homoduplexes, owing to its nonionic nature. ${ }^{[7]}$ When it comes to in vivo application, a significant drawback regarding PNAs as biomolecular tools arises from their inadequate membrane permeability.

An effective solution to the above critical problem requires the development of structurally distinct oligomers. Up to now, few independent literature reports described autonomous pairing systems builded upon $\mathrm{N}$-(2-hydroxyethyl)phosphonoglycine $(\mathrm{O}$ pPNAs) or $N$-(2-aminoethyl)phosphonoglycine ( $N$-pPNAs) backbones (Figure 1). ${ }^{[8-12]}$ Although such family of mimics benefits from the insertion of a phosphono-ester unit in their backbones, which gives them a better metabolic stability, they display

[a] Departamento de Química, Universidade Federal Rural de Pernambuco, 52171-900 Recife, PE, Brasil

[b] Laboratory for Medicinal Chemistry, Rega Institute for Medical Research, KU Leuven, Minderbroedersstraat 10, 3000 Leuven, Belgium

E-mail: Piet.Herdewijn@rega.kuleuven.be

Homepage: http://medchem.rega.kuleuven.ac.be/

Supporting information for this article is available on the WWW

under http://dx.doi.org/10.1002/ejoc.xxxxxxxxx. considerably weaker hybridization affinity for cognate DNA and RNA as compared to PNAs and natural oligonucleotide counterparts. A certain improved ability for heteroduplex formation resulted from chimeric sequences alternating pPNA and PNA/PNA-like monomers. ${ }^{[13-15]}$

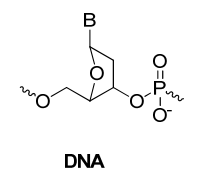<smiles>[R]C(C(C)=O)N(CC(C)NC)C([B])=O</smiles><smiles>[B]C(=O)N(CCNC)CC(C)=O</smiles><smiles>[B]C(=O)N(CCNC)C[P+](C)(C)[O-]</smiles>

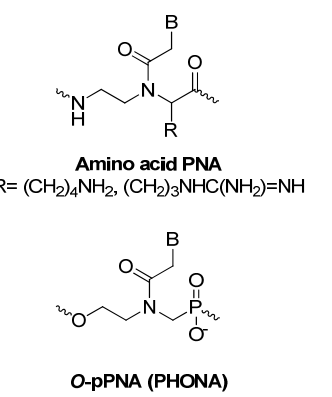

O-PPNA (PHONA)
Figure 1. Repeating monomeric units of DNA, PNA and its closely related DNA mimics (selected examples).

Given our long-standing interest in the search for novel artificial oligonucleotides, we set out to design and synthesize modified phosphono building blocks 1a-c and $\mathbf{2}$ (Figure 2) as precursors to a new generation of polymers. We are investigating the possibility that a supplementary hydroxyl group in the backbone of pPNAs might facilitate groove solvation and thus prove to be advantageous upon binding with DNA and/or RNA targets. In the proposed structures, the internucleotide linkers contain 7 or 6 bonds dependent on which hydroxyl group is used for ester formation, and both are compatible with hybridization properties. ${ }^{[16]}$ Here, we present our synthetic efforts to the preparation of achiral and chiral phosphonoglycol monomers depicted in Figure 2.

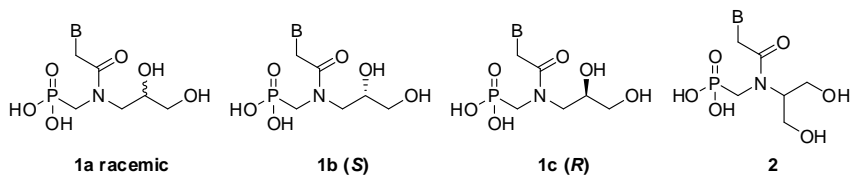

Figure 2. General chemical structures of planned monomeric targets. 


\section{Results and Discussion}

From a strategic point of view, it was first decided that the target building blocks 1a-c and 2 should be assembled by stepwise addition of phosphonate and nucleobase moieties to appropriately protected amino dihydroxy synthons. Preliminary efforts were directed towards the preparation of the key racemic glycol derivative 6, which would serve as precursor for 1a (Scheme 1). Thus, selective $N$-benzoylation ${ }^{[17,18]}$ of 3-amino-1,2-dihydroxy propane 3 , followed by tritylation of compound 4 , generated secondary alcohol $\mathbf{5}$, which, upon harsh basic hydrolysis, afforded 3-amino-1-O-trityl-2-propanol 6 in good overall yield.

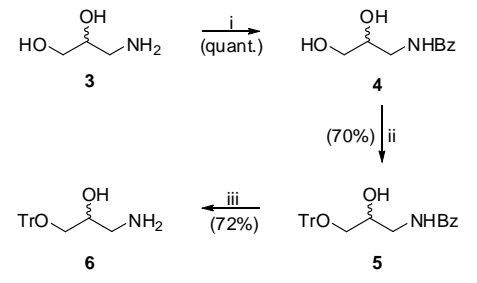

Scheme 1. Reagents and conditions: i) $\mathrm{BzCl}, \mathrm{K}_{2} \mathrm{CO}_{3}, \mathrm{H}_{2} \mathrm{O}$. ii) $\mathrm{TrCl}$, Py. iii) $4 \mathrm{~N}$ aq. $\mathrm{NaOH}, \mathrm{EtOH}, \Delta$.

Next, in order to obtain both enantiomeric forms of the above aminoalcohol, different routes were explored. The known $(S)-1-O$ trityl-2,3-propanediol 7, previously obtained from 5-O-trityl- $(D)$ arabinofuranose, ${ }^{[19]}$ was our initially chosen substrate. However, conventional tosylation of 7 proceeded with surprisingly little selectivity, and a mixture of the desired 3-O-tosylate $8(54 \%)$ and 2,3-bis-O-tosylate 9 (23\%) was formed (Scheme 2). More importantly, 8 and $\mathbf{9}$ were difficult to separate by chromatography due to very similar $R_{f} s$. In view of those difficulties, we resorted to the tin methodology. ${ }^{[20]}$ Treatment of diol 7 with $\mathrm{Bu}_{2} \mathrm{SnO}$ followed by $\mathrm{TsCl}$, permitted isolation of the required monotosylate $\mathbf{8}$ in $97 \%$ yield over two steps.

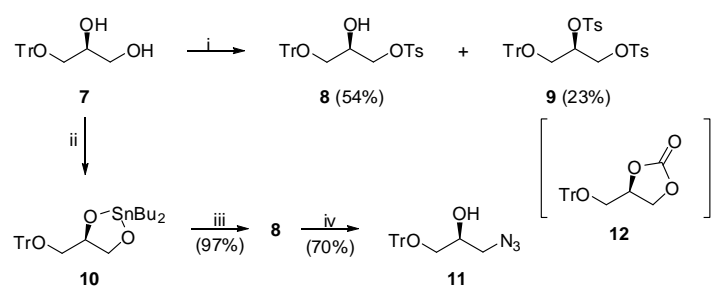

Scheme 2. Reagents and conditions: i) TsCl, Py. ii) $\mathrm{Bu}_{2} \mathrm{SnO}, \mathrm{MeOH}$. iii) TsCl, THF. iv) $\mathrm{NaN}_{3}$, DMF.

Subsequent substitution using $\mathrm{NaN}_{3}$ furnished chiral azide $(S)$-11 in $70 \%$ yield, while the methodology described by Kutney et al. ${ }^{[21]}$ via transient cyclic carbonate $\mathbf{1 2}$ or Mitsunobu reaction ${ }^{[22]}$ using $\mathbf{7}$ and diisopropylazodicarboxylate/ $\mathrm{Ph}_{3} \mathrm{P} / \mathrm{HN}_{3}$ were both unsuccessful. Both compounds $\mathbf{6}$ and $\mathbf{1 1}$ were abandoned later due to difficulties during a reduction of the azide function, during a phosphonylation step and subsequent coupling with thymin-1-yl acetic acid (see below).

As an alternative route, we decided to turn to commercially available $(R)$-glycidol 13 as precursor to our desired chiral synthon.
After silylation of $\mathbf{1 3}$ at the primary position, ${ }^{[23]}$ the resulting protected epoxide derivative $\mathbf{1 4}$ (which can also be obtained by kinetic resolution ${ }^{[24]}$ or by asymmetric epoxidation ${ }^{[25]}$ ) underwent noticeable decomposition during its ring opening to azido alcohol 15 (Scheme 3) and only marginal quantity of the expected compound 15 (ca 3\%) could be isolated. Pleasingly, the replacement of $\mathbf{1 3}$ with $(S)$-1,2-isopropylideneglycerol (S-Solketal) 16 as starting material led to much improved results in terms of isolated yields. Compound $\mathbf{1 6}$ and its enantiomer are produced in ton scale by dessymetrization of 1,2- isopropylideneglycerol hydrogen phthalate using chiral 1-phenylethylamine. ${ }^{[26]}$

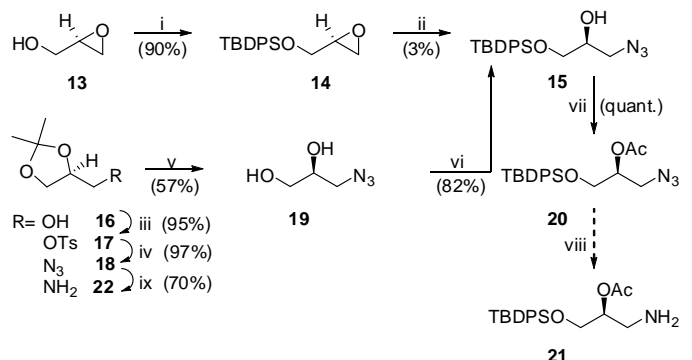

Scheme 3. Reagents and conditions: i) TBDPSCl, $\mathrm{Et}_{3} \mathrm{~N}, \mathrm{CH}_{2} \mathrm{Cl}_{2}$. ii) $\mathrm{NaN}_{3}$, DMF, $78{ }^{\circ} \mathrm{C}$. iii) $\mathrm{TsCl}$, Py. iv) $\mathrm{NaN}_{3}$, DMF. v) pTSA $\cdot \mathrm{H}_{2} \mathrm{O}, \mathrm{MeOH}$. vi) TBDPSCl, Im, DMF. vii) $\mathrm{Ac}_{2} \mathrm{O}$, Py. viii) $\mathrm{Ph}_{3} \mathrm{P}, \mathrm{H}_{2} \mathrm{O}$. ix) $\mathrm{Ph}_{3} \mathrm{P}, \mathrm{THO}, \mathrm{H}_{2} \mathrm{O}$ or $\mathrm{Na}_{2} \mathrm{~S} \cdot 9 \mathrm{H}_{2} \mathrm{O}$.

Following tosylation of the hydroxyl group on $\mathbf{1 6}^{[27]}$ and substitution using $\mathrm{NaN}_{3},{ }^{[28]}$ hydrolysis of the isopropylidene protection upon treatment with $p \mathrm{TSA} \cdot \mathrm{H}_{2} \mathrm{O}^{[28,29]}$ and selective silylation at the primary hydroxyl group generated the desired $(S)$ 3-azido-1-t-butyldiphenylsilyloxy-2-propanol $\mathbf{1 5}$ in good yield $(82 \%)$. This material was identical to that prepared through the previous approach. Acetylation of $\mathbf{1 5}$ proceeded quantitatively, but attempted conversion of the azide into the required amino function to give 21 via Staudinger reaction ${ }^{[30]}\left(\mathrm{Ph}_{3} \mathrm{P}\right.$, wet THF) resulted in a mixture of products presumably resulting from migration of the acetyl group and possibly of the silyl group as well. Consequently, compound $\mathbf{2 0}$ as a precursor of $\mathbf{1 b}$ was abandoned. Evidently, a non-migrating hydroxyl-protecting group in $\mathbf{1 5}$, as well as in 11, like a benzyl group, must be used. This remains to be investigated. In light of these results, we decided to use $(S)$-azide $\mathbf{1 8}$ directly as such instead of transforming it into hydrophobic compound 20. Treatment of 18 with either $\mathrm{Na}_{2} \mathrm{~S} \cdot 9 \mathrm{H}_{2} \mathrm{O}^{[31]}$ or more conveniently under Staudinger reaction conditions ${ }^{[30]}$ accomplished the reduction of the azido function, affording the corresponding amine $(S)-22^{[32,33]}$ in $70 \%$ isolated yield in both cases. The amine 22 is very polar and inconvenient to handle on a small scale. Its advantage however consists in the liberation of both hydroxyl groups in a single deprotection step (see below).

Although the corresponding (R)-3-amino-1,2-O-isopropylidenoxy propane, could be obtained by repeating the same synthetic sequence shown in Scheme 3, starting from readily available enantiomeric $(R)$-Solketal, ${ }^{[26]}$ we devised a different approach based on $(D)$-galactose (Scheme 4$)$. (D)-Galactose is the only easily available sugar that can be converted in one step into its derivative 23, which exposes a primary hydroxyl group. We reasoned that this compound could be transformed into a 6-amino6-deoxy- $(D)$-galactopyranose derivative $\mathbf{2 8}$, which in turn could be degraded by $\mathrm{NaIO}_{4}$ to form $(R)$ amine 29 in which the stereogenic atom $\mathrm{C} 2$ would be the $\mathrm{C} 5$ atom in its predecessor 27. Galactose does not seem to be much exploited in this sense. ${ }^{[34]}$ 


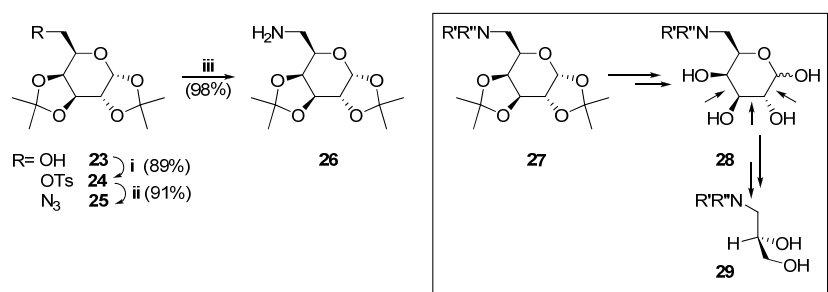

Scheme 4. Reagents and conditions: i) $\mathrm{TsCl}, \mathrm{Py}$. ii) $\mathrm{NaN}_{3}, \mathrm{DMF}, 120{ }^{\circ} \mathrm{C}$. iii) $\mathrm{Ph}_{3} \mathrm{P}, \mathrm{THF}, \mathrm{H}_{2} \mathrm{O}$.

The logic of this procedure in terms of "economy of. chirality" can be questioned since only one stereogenic center present in the substrate $\mathbf{2 7}$ is used, and three others are lost. Nevertheless, ease of accessibility to 1,2;3,4-di- $O$-isopropylidene- $(D)$-galactopyranose $\mathbf{2 3}^{[35]}$ prompted us to use it as described above. Commercially available 23 was converted into its 6-O-tosylate $24^{[35]}$ which after treatment with an excess of $\mathrm{NaN}_{3}$ furnished azide 25. ${ }^{[36]}$ This apparently simple substitution at the primary position of $\mathbf{2 4}$ is known to require extended reaction time, elevated temperatures and excess of nucleophile. The use of a triflate derived from 23 rather than tosylate $\mathbf{2 4}$ makes substitutions much easier. ${ }^{[37-39]}$ The azide $\mathbf{2 5}$ was reduced to the amine $\mathbf{2 6}$ in nearly quantitative yield using again Staudinger conditions. The same compound was previously obtained using catalytic hydrogenation ${ }^{[36]}$ of 25 or lithium aluminum hydride reduction of the corresponding 6oxime. ${ }^{[40]}$

The last amine necessary for the synthesis of achiral building block 2 was easily obtained by standard silylation of 2-amino-1,3propanediol (Scheme 5). An unsignificant amount of monosilylated compound 32 was also formed together with the desired 2-amino1,3-bis-t-butydiphenylsilyloxy propane $\mathbf{3 1}$.

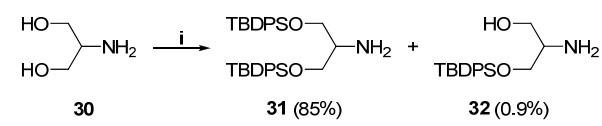

Scheme 5. Reagents and conditions: i) TBDPSCl, Im, DMF.

Having successfully obtained the desired protected amines 6, 22, $\mathbf{2 6}$ and $\mathbf{3 1}$ in workable quantities, the subsequent transformation for installing the phosphonate group was achieved using standard alkylation conditions, as illustrated in Table 1 .

The required alkylating reagents $33 a^{[41]}\left(\mathrm{R}^{\prime}=\mathrm{SO}_{2} \mathrm{C}_{6} \mathrm{H}_{4} \mathrm{CH}_{3}\right)$ and 33b $\mathbf{b}^{[42]}\left(\mathrm{R}^{\prime}=\mathrm{SO}_{2} \mathrm{CF}_{3}\right)$ were prepared according to literature procedures. The reaction of diethylphosphonomethyl tosylate 33a with primary amines $\mathbf{2 2}$ and $\mathbf{2 6}$ gave phosphonomethylamines $\mathbf{3 4}$ and 35 in moderate yields ( 20 to $30 \%$ ), while the amine 31 underwent alkylation with more reactive triflate $\mathbf{3 3 b}$ in good yield $(\sim 56 \%)$. Phosphonylation of racemic amine 6 with 33b was possible, but this reaction was complicated by facile double alkylation at $\mathrm{NH}_{2}$, which led to mono- $N$-alkylated derivative 37 $(18 \%)$ together with $22 \%$ of doubly phosphonomethylated product 38. Consequently, it proved necessary to turn to another substrate, such as commercially available 3-amino-1,2-isopropylidenoxy propane 39, which gave $30 \%$ yield of alkylated product. It should be mentioned that attempts to increase yields by using diethylphosphonomethylsulfonoimidazole in the place of 33a or 33b were uniformly unsuccessful. The sulfonoimidazole moiety was claimed to be a better leaving group than a tosylate/mesylate but inferior to triflate, ${ }^{[43]}$ and can be obtained is situ by reacting $(\mathrm{EtO})_{2} \mathrm{P}(\mathrm{O}) \mathrm{CH}_{2} \mathrm{OH}$ with sulfuryl diimidazole ${ }^{[44]}$ and $\mathrm{NaH}$, or with sulfuryl chloride and imidazole. ${ }^{[43]}$ Unexpectedly, subsequent addition of amine produced no traces of the desired products.

Table 1. Synthesis of phosphonomethylamines 34-38, 40

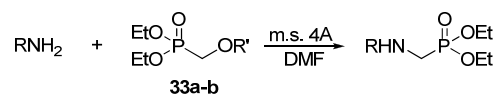

entry amine

In the following step, phosphonomethylamines 34, 35, 36 and 40 were reacted in the presence of DCC and hydroxybenzotriazole with thymin-1-yl acetic acid $\mathbf{4 1}$ (Scheme 6), obtained in turn by alkylation of thymine with chloroacetic acid in basic medium. ${ }^{[45]}$ The corresponding amides were generally produced in excellent yields, the only exception to this trend being the amide derived from amine 36, most probably due to the steric hindrance of the bulky silyl goups.

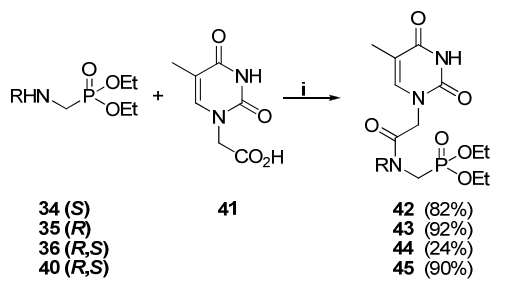

Scheme 6. Reagents and conditions: i) DCC, HOBt, DMF.

At this point, compound $\mathbf{4 3}$ was subjected to further manipulation via the deprotection-degradation sequence discussed earlier (Scheme 7). First, the acetonide protecting groups were removed by exposure to aqueous TFA, leading to tetraol 46 in $86 \%$ yield. Next, this compound was reacted with four equivalents of sodium periodate under carefully controlled conditions. We recognized that the resulting transient aldehyde would be prone to fast decomposition, therefore it was immediately reduced upon treatment with sodium borohydride. Gratifyingly, the desired diol 47 could be isolated in reasonable yield (52\%), together with minor amounts of two degradation fragments, presumably formed in the initial oxidation step. The reaction that concluded our synthesis 
was a dealkylation using trimethylsilyl iodide (TMSI) and furnished the $(R)$-monomer $\mathbf{5 0}$, a precursor of $\mathbf{1 c}$.

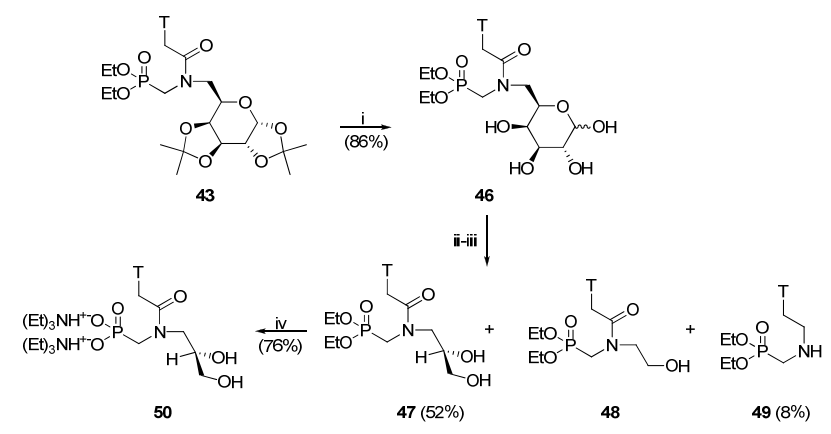

Scheme 7. Reagents and conditions: i) $80 \%$ aq.TFA. ii) $\mathrm{NaIO}_{4}, \mathrm{EtOH}, \mathrm{H}_{2} \mathrm{O}$ iii) $\mathrm{NaBH}_{4}, \mathrm{EtOH}$. iv) TMSI, DMF; ion exchange chromatography (1M TEAB: $\mathrm{H}_{2} \mathrm{O}$ ).

TMSI acting in turn on compounds 42 and 45, allowed removal of the isopropylidene acetal and cleavage of the diethyloxy ether groups in a single synthetic operation, as depicted in Scheme 8 . Purification on silica gel, followed by anion exchange chromatography completed access to phosphonates $\mathbf{5 1}$ and 52, precursors of $\mathbf{1 b}$ and $\mathbf{1 a}$ respectively.

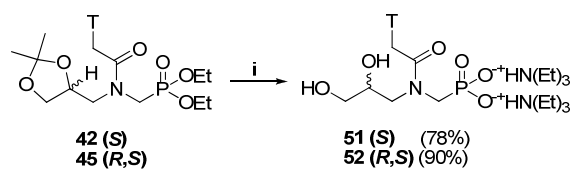

Scheme 8. Reagents and conditions: i) TMSI, Et ${ }_{3} \mathrm{~N}, \mathrm{DMF}$; ion exchange chromatography (1M TEAB: $\left.\mathrm{H}_{2} \mathrm{O}\right)$.

Bis-silylated phosphonamido thymine $\mathbf{4 4}$ was initially treated with TBAF to effect liberation of the hydroxyl functionalities. Unfortunately, this approach invariably provided a complex mixture of inseparable products, most likely due to the sensitivity of the substrate to a basic media. The same outcome was obtained using $\mathrm{HF} /$ pyridine complex. After those unsuccessful attempts, we found that the use of a milder reagent such as tris(dimethylamino)sulfonium difluorotrimethylsilicate (TAS-F) ${ }^{[46]}$ was critical to avoid formation of undesired by-products, mainly arising from preferential formation of cyclic phosphonates. Finally, the phosphono functionality of $\mathbf{5 3}$ was deprotected by the action of TMSI (Scheme 8) to give monomer 54.

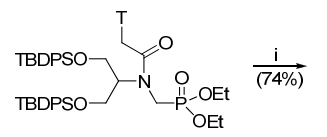

44

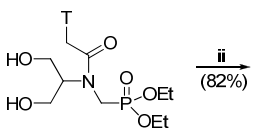

53

Scheme 9. Reagents and conditions: i) TAS-F, DMF, $0{ }^{\circ} \mathrm{C}$ to rt. ii) TMSI, $\mathrm{Et}_{3} \mathrm{~N}$, DMF; ion exchange chromatography (1M TEAB: $\left.\mathrm{H}_{2} \mathrm{O}\right)$.

\section{Conclusions}

In summary, synthetic routes have been developed for the preparation of new backbone motifs, comprising of $\mathrm{N}$-alkylated phosphonoglycine moieties. These monomers will be used for constructing new unnatural nucleic acids with a phosphonate internucleotide linkage.

\section{Experimental Section}

General methods. NMR spectra were recorded on Bruker Avance $300(1 \mathrm{H} 300 \mathrm{MHz}, 13 \mathrm{C} 75 \mathrm{MHz}, 31 \mathrm{P} 121 \mathrm{MHz})$ or Avance II 500 (1H $500 \mathrm{MHz}, 13 \mathrm{C} 125 \mathrm{MHz}, 31 \mathrm{P} 202 \mathrm{MHz})$ spectrometers. Spectra were referenced to TMS and chemical-shift $(\delta)$ values, expressed in parts per million (ppm), are reported downfield of TMS. The multiplicity of signals is reported as singlet (s), doublet (d), triplet (t), quartet (q), multiplet (m), broad (br) or a combination of any of these. Coupling constants are expressed in hertz $(\mathrm{Hz})$. For final products, $1 \mathrm{H}$ and $13 \mathrm{C}$ resonance assignments were made with the aid of $1 \mathrm{H}-1 \mathrm{H}$ and $1 \mathrm{H}-13 \mathrm{C}$ correlation experiments. High resolution mass spectra (HRMS) were acquired on a quadrupole orthogonal acceleration time-of-flight mass spectrometer (Synapt G2 HDMS, Waters, Milford, MA), utilizing electrospray ionization (ESI). Samples were infused at $3 \mathrm{uL} / \mathrm{min}$ and spectra were obtained in positive (or negative) ionization mode with a resolution of 15000 (FWHM) using leucine enkephalin as lock mass. Chromatographic separations were performed with silica gel (100-200 mesh or 230-400 mesh) and analytical thinlayer chromatography was performed on precoated aluminum backed sheets $(254 \mathrm{~nm})$ supplied by Fluka. For all reactions, analytical grade solvents were used. Anhydrous THF was refluxed over sodium-benzophenone ketyl and distilled. Dry dichloromethane was obtained by distillation over $\mathrm{CaH}_{2}$ and dry pyridine was refluxed and stored over KOH. Dry dimethylformamide was supplied by Aldrich. All final compounds were purified by anion exchange chromatography (Source 15Q), using $\mathrm{H}_{2} \mathrm{O}$ and $1 \mathrm{M}$ TEAB as eluents. Optical rotations were recorded on a Perkin-Elmer 341 polarimeter in a thermostated cell of length $1 \mathrm{dm}$ at $20{ }^{\circ} \mathrm{C}$ using the sodium D-line, and a suitable solvent that is reported along with the concentration (in $\mathrm{g} / 100 \mathrm{ml}$ ).

(Rac)-3- $N$-Benzamido-1-triphenylmethyloxy-2-propanol (5): To a cold (ice-water bath) solution of 3-amino-1,2-propane diol (9.1 g, $100 \mathrm{mmol})$ in $\mathrm{H}_{2} \mathrm{O}(70 \mathrm{ml})$ was added $\mathrm{K}_{2} \mathrm{CO}_{3}(30 \mathrm{~g})$ followed by benzoyl chloride $(13.5 \mathrm{ml}, 16.3 \mathrm{~g}, 116 \mathrm{mmol})$ dropwise. The mixture was then magnetically stirred overnight at $40{ }^{\circ} \mathrm{C}$. The product 4 has $\mathrm{R}_{\mathrm{f}} 0.36$ in $\mathrm{CH}_{2} \mathrm{Cl}_{2}-\mathrm{MeOH}$ 10:0.7 (staining with ninhydrin). The reaction mixture was quenched by addition of $1 \mathrm{M}$ $\mathrm{HCl}$ and subsequently evaporated to dryness. The resulting residue was resuspended in ethanol, the mixture was then filtered and the filtrate was evaporated under reduced pressure. Chromatography in $\mathrm{CH}_{2} \mathrm{Cl}_{2}-\mathrm{MeOH}$ (gradient) 10:0.7 $\rightarrow$ 10:1 furnished 4 (19.4 g, quantitatively) as a colorless syrup. ${ }^{[17,18]}$ The NMR data match those published before. ${ }^{[18]}$ This compound $(20.0 \mathrm{~g}, 102 \mathrm{mmol})$ in dry $\mathrm{CH}_{2} \mathrm{Cl}_{2}(100 \mathrm{ml})$ and pyridine $(35 \mathrm{ml})$ was cooled in an icewater bath under argon atmosphere, and $\mathrm{TrCl}(32.0 \mathrm{~g}, 115 \mathrm{mmol})$ was added in one portion while maintaining magnetic stirring. Cooling was removed and the reaction mixture was left overnight at r.t., followed by $48 \mathrm{~h}$ at gentle boiling. TLC showed the product 5 Rf 0.55 in hexane-EtOAc $1: 1$, yellow using $\mathrm{CrO}_{3}-\mathrm{H}_{2} \mathrm{SO}_{4}$ system, or pink using ninhydrin. Extraction $\left(\mathrm{CH}_{2} \mathrm{Cl}_{2}-\mathrm{H}_{2} \mathrm{O}\right)$, drying, evaporation and coevaporation with xylenes, resulted in spontaneous crystallization. The crystals were filtered, washed with hexane and dried to yield 5 (31.5 g, 70\%), m.p. $170-174{ }^{\circ} \mathrm{C} .{ }^{1} \mathrm{H}$ NMR (300 MHz, DMSO- $d 6): \delta=8.33(\mathrm{t}, J=5.5 \mathrm{~Hz}$, exchangeable, $1 \mathrm{H}), 7.29(\mathrm{~d}, J=6.9 \mathrm{~Hz}, 2 \mathrm{H}), 7.77-7.21(\mathrm{~m}$, aromatic, $18 \mathrm{H}), 5.12$ $(\mathrm{d}, J=5.3 \mathrm{~Hz}$, exchangeable, $1 \mathrm{H}$ ), 3.92 (m of 6 lines, $J=5.6 \mathrm{~Hz}, 1$ $\mathrm{H}$ ), 3.53-3.45 and 3.29-3.20 (two m, $1 \mathrm{H}$ each), 3.01 (dd, $J=5.5$, $9.5 \mathrm{~Hz}, 1 \mathrm{H}), 2.97(\mathrm{dd}, J=5.0,9.0 \mathrm{~Hz}, 1 \mathrm{H}) \mathrm{ppm}$. After $\mathrm{D}_{2} \mathrm{O}$ exchange: $\delta=3.91$ (m of 5 lines, $J=5.7 \mathrm{~Hz}$ ), 3.37 (dd, $J=5.3$, $13.4 \mathrm{~Hz}), 3.25(\mathrm{dd}, J=7.1,13.3 \mathrm{~Hz}) \mathrm{ppm} .{ }^{13} \mathrm{C} \mathrm{NMR}(75 \mathrm{MHz}$, DMSO-d6): $\delta=166.4,143.9,134.5,131.0,128.3,128.1,127.8$, 127.2, 126.9, 85.8, 68.5, 66.2, 45.4 ppm. HRMS (pos.): calcd. for $\mathrm{C}_{29} \mathrm{H}_{27} \mathrm{NNaO}_{3}[\mathrm{M}+\mathrm{Na}]^{+} 460.1883$; found 460.1876 . 
(Rac)-3-Amino-1-triphenylmethyloxy-2-propanol

(6):

Compound $5(2.0 \mathrm{~g}, 4.5 \mathrm{mmol})$ in EtOH $(40 \mathrm{ml})$ and $4 \mathrm{~N} \mathrm{NaOH}(30$ $\mathrm{ml})$ was stirred under reflux during $48 \mathrm{~h}$. Water $(60 \mathrm{ml})$ was added and the resulting heterogeneous mixture was kept in ice-water for 1 h. Precipitated colorless material was filtered, washed with icewater, dried on oil pump and solubilized in EtOH $(50 \mathrm{ml})$ with warming. Some solid material was removed by filtration and volatiles were evaporated. TLC showed a spot of the product $\mathrm{R}_{\mathrm{f}}$ $0.40 \quad\left(\mathrm{CH}_{2} \mathrm{Cl}_{2}-\mathrm{MeOH}-25 \% \quad \mathrm{NH} 4 \mathrm{OH} \quad\right.$ 20:2:0.1), purple with ninhydrin or yellow with Hanessian's stain. Chromatography in the same system furnished $6(1.1 \mathrm{~g}, 72 \%)$ as a glassy material. Addition of $\mathrm{Et}_{2} \mathrm{O}$ resulted in the formation of a colorless powder that has a melting range $c a 77^{\circ}-c a 115{ }^{\circ} \mathrm{C} .{ }^{1} \mathrm{H}$ NMR $(300 \mathrm{MHz}$, DMSO-d6): $\delta=7.45-7.22(\mathrm{~m}$, aromatic, $15 \mathrm{H}), 3.67-3.59(\mathrm{~m}$ unresolved, $1 \mathrm{H}), 3.01(\mathrm{dd}, J=5.5,9.0 \mathrm{~Hz}, 1 \mathrm{H}), 2.90(\mathrm{dd}, J=6.0$, $9.0 \mathrm{~Hz}, 1 \mathrm{H}), 2.71(\mathrm{dd}, J=4.1,12.7 \mathrm{~Hz}, 1 \mathrm{H}), 2.51(\mathrm{dd}, J=7.1$, $12.6 \mathrm{~Hz}, 1 \mathrm{H})$ ppm. ${ }^{13} \mathrm{C}$ NMR $(75 \mathrm{MHz}$, DMSO-d6): $\delta=143.9$, $128.3,127.8,126.9,85.7,71.1,65.9,45.3$ ppm. HRMS (pos.): calcd. for $\mathrm{C}_{22} \mathrm{H}_{24} \mathrm{NO}_{3}[\mathrm{M}+\mathrm{H}]^{+} 334.1801$; found 334.1819 .

(R)-1-Tosyloxy-3-triphenylmethyloxy-2-propanol (8) and (R)1,2-bis-tosyloxy-3-triphenylmethyloxy propane (9): (S)-Diol $7^{[19]}(0.25 \mathrm{~g}, 0.75 \mathrm{mmol})$ in dry $\mathrm{CH}_{2} \mathrm{Cl}_{2}(15 \mathrm{ml})$ and $\mathrm{Et}_{3} \mathrm{~N}(0.3 \mathrm{ml})$ was treated with $\mathrm{TsCl}(0.19 \mathrm{~g}, 1 \mathrm{mmol})$ during $18 \mathrm{~h}$ under protective atmosphere (argon). TLC showed two closely positioned compounds ( $\mathrm{R}_{\mathrm{f}}$ of a mid-point 0.38 in hexane-EtOAc 140:55). Extraction $\left(\mathrm{CH}_{2} \mathrm{Cl}_{2}-\mathrm{H}_{2} \mathrm{O}\right)$, drying $\left(\mathrm{MgSO}_{4}\right)$, evaporation and flash chromatography yielded $9(0.11 \mathrm{~g}, 23 \%)$ and $8(0.197 \mathrm{~g}, 54 \%)$. Data for 9: foam, $\alpha_{D}=-2.1^{\circ}$ c 3.2 dioxane. ${ }^{1} \mathrm{H}$ NMR $(300 \mathrm{MHz}$, $\left.\mathrm{CDCl}_{3}\right): \delta=7.70-7.60(\mathrm{~m}$, aromatic, $4 \mathrm{H}), 7.25(\mathrm{~m}$, aromatic, $15 \mathrm{H})$ 4.55 (m of 5 lines, $J=4.9 \mathrm{~Hz}, 1 \mathrm{H}$ ), 4.15-4.13 (m unresolved, $2 \mathrm{H}$ ), $3.28(\mathrm{dd}, J=5.2,10.0 \mathrm{~Hz}, 1 \mathrm{H}), 3.23(\mathrm{dd}, J=4.9,10.0 \mathrm{~Hz}, 1 \mathrm{H})$, 2.43 (s, $6 \mathrm{H})$ ppm. ${ }^{13} \mathrm{C} \mathrm{NMR}\left(75 \mathrm{MHz}, \mathrm{CDCl}_{3}\right): \delta=145.3,145.2$, $143.3,133.3,132.4,130.1,130.0,128.7,128.2,128.2,128.1,127.4$ $87.4,77.5,67.8,62.1,21.9$ ppm. HRMS (pos.): calcd. for $\mathrm{C}_{36} \mathrm{H}_{34} \mathrm{NaO}_{7} \mathrm{~S}_{2}[\mathrm{M}+\mathrm{Na}]^{+}$665.1638; found 665.1634. Data for 8 foam, $\alpha_{D}=-11.3^{\circ}$ c 1.8 dioxane. ${ }^{1} \mathrm{H}$ NMR $\left(300 \mathrm{MHz}, \mathrm{CDCl}_{3}\right): \delta=$ $7.76(\mathrm{~d}, J=8.2 \mathrm{~Hz}, 2 \mathrm{H}), 7.37-7.22(\mathrm{~m}, 17 \mathrm{H}), 4.15(\mathrm{dd}, J=4.4$ $10.2 \mathrm{~Hz}, 1 \mathrm{H}), 4.06(\mathrm{dd}, J=6.0,10.1 \mathrm{~Hz}, 1 \mathrm{H}), 3.92$ (m of 6 lines, $J$ $=5.2 \mathrm{~Hz}, 1 \mathrm{H}), 3.21(\mathrm{dd}, J=5.3,9.7 \mathrm{~Hz}, 1 \mathrm{H}), 3.16(\mathrm{dd}, J=5.5$, $9.8 \mathrm{~Hz}, 1 \mathrm{H}), 2.42(\mathrm{~s}, 3 \mathrm{H}), 2.35(\mathrm{~d}, J=5.6 \mathrm{~Hz}, 1 \mathrm{H}) \mathrm{ppm} .{ }^{13} \mathrm{C}$ NMR $\left(75 \mathrm{MHz}, \mathrm{CDCl}_{3}\right): \delta=145.2,143.6,132.8,130.1,128.7$, $128.2,128.1,127.4,87.1,71.2,68.9,63.8,21.8$ ppm. HRMS (pos.): calcd. for $\mathrm{C}_{29} \mathrm{H}_{28} \mathrm{NaO}_{5} \mathrm{~S} \quad[\mathrm{M}+\mathrm{Na}]^{+}$511.1549; found 511.1554 .

Compound 8 via tin oxide methodology ${ }^{[20]}$ : Diol 7 (0.24 g, 0.71 $\mathrm{mmol})$ in dry toluene $(20 \mathrm{ml})$ and dibutyltin oxide $(0.23 \mathrm{~g}, 0.92$ $\mathrm{mmol}$ ) were magnetically stirred at gentle reflux under argon. The mixture became homogenous after $c a 20 \mathrm{~min}$. After a total of 80 min the mixture was cooled down, evaporated and dried on oil pump. The vacuum was broken using dry argon in a balloon. To product 10 thus obtained was added dry THF $(10 \mathrm{ml})$ followed by $\mathrm{TsCl}(0.15 \mathrm{~g}, 0.8 \mathrm{mmol})$, and this mixture was left overnight at $\mathrm{rt}$. TLC showed the monotosylate 8 as the only product. Evaporation of the solvent and flash chromatography in hexane-EtOAc 7:3 furnished 8 (0.25 $\mathrm{g}, 97 \%)$.

(S)-1-Azido-3-triphenylmethyloxy-2-propanol (11): Tosylate 8 $(0.13 \mathrm{~g}, 0.26 \mathrm{mmol})$ in dry DMF $(10 \mathrm{ml})$ and $\mathrm{NaN}_{3}(0.65 \mathrm{~g}, 10$ mmol) were stirred under argon at $88{ }^{\circ} \mathrm{C}$ for $2.5 \mathrm{~h}$. TLC showed new intensely charring spot (Hanessian's stain) less polar than a substrate $R_{f}$ 0.64, hexane-EtOAc 7:3 (substrate's $R_{f}$ is 0.40 ). Extraction $\left(\mathrm{CH}_{2} \mathrm{Cl}_{2}-\mathrm{H}_{2} \mathrm{O}\right)$, drying $\left(\mathrm{MgSO}_{4}\right)$, filtration, evaporation and purification by preparative TLC plate using hexane-EtOAc $4: 1$, furnished glassy product $(0.067 \mathrm{~g}, 70 \%) . \alpha_{D}=-8.4^{\circ}$, c $3.4 \mathrm{CHCl}_{3} .{ }^{1} \mathrm{H}$ $\operatorname{NMR}\left(300 \mathrm{MHz}, \mathrm{CDCl}_{3}\right): \delta=7.42-7.23(\mathrm{~m}$, aromatic, $15 \mathrm{H}), 3.88$ (unresolved t, $J=5.3 \mathrm{~Hz}, 1 \mathrm{H}), 3.35(\mathrm{~d}, J=5.3 \mathrm{~Hz}, 2 \mathrm{H}), 3.20(\mathrm{~d}, J$ $=5.3 \mathrm{~Hz}, 2 \mathrm{H}), 2.39$ (br s, $1 \mathrm{H}) \mathrm{ppm} .{ }^{13} \mathrm{C} \mathrm{NMR}\left(75 \mathrm{MHz}, \mathrm{CDCl}_{3}\right)$ : $\delta=143.5,128.5,127.9,127.2,86.9,70.1,64.7,53.8$ ppm. HRMS (pos.): calcd. for $\mathrm{C}_{22} \mathrm{H}_{21} \mathrm{~N}_{3} \mathrm{NaO}_{2} \quad[\mathrm{M}+\mathrm{Na}]^{+} 382.1526$; found 382.1532. Attempts to obtain the azide $\mathbf{1 1}$ via a cyclic carbonate $\mathbf{1 2}$ by analogy to the other substrates ${ }^{[21]}$ (diol 7 and carbonyldiimidazole, followed by $\mathrm{NaN}_{3}$ ) or using a Mitsunobu reaction ${ }^{[22]}\left(7, \mathrm{PPh}_{3}, \mathrm{HN}_{3}, i \mathrm{PrO}_{2} \mathrm{CNNCO}_{2} i \mathrm{Pr}\right)$ were negative.

(S)-3-Azido-1-tbutyldiphenylsilyloxy-2-propanol (15): Method a. Starting from $(R)$-glycidol: $(R)$-glycidol (Sigma-Aldrich) 13 was conventionally silylated ${ }^{[23]}$ to furnish $\mathbf{1 4}$ as an oil in ca $90 \%$ yield after chromatography (gradient, hexane-Et $\mathrm{H}_{2} \mathrm{O} \quad 10: 0.5 \rightarrow 10: 1.5$ ), $\mathrm{R}_{\mathrm{f}}$ 0.47 in hexane-Et $2 \mathrm{O} 10: 1, \alpha_{D}-1.9^{\circ}$ c $21.6, \mathrm{CHCl}_{3}$, lit. $\alpha_{D}-1.4^{\circ}$ c 1 $\mathrm{CHCl}_{3},{ }^{[24]} \alpha_{D}-2.3^{\circ}$ c $9.07 \mathrm{CHCl}_{3} .^{[25]}$ (Optical rotation is not presented in the ref. 13).This compound $(1.21 \mathrm{~g}, 3.9 \mathrm{mmol})$ in dry DMF $(24 \mathrm{ml})$ and $\mathrm{NaN}_{3}(2.0 \mathrm{~g}, 30 \mathrm{mmol})$ was stirred under argon at $78{ }^{\circ} \mathrm{C}$ during $8 \mathrm{~h}$. The mixture turned brown. TLC showed the product $15 \mathrm{R}_{\mathrm{f}} 0.34$ in hexane-Et $\mathrm{t}_{2} \mathrm{O} \quad 4: 1$, along with the decomposition products. Extraction $\left(\mathrm{CH}_{2} \mathrm{Cl}_{2}\right.$-water $)$, drying, evaporation of solvent and chromatography (gradient, hexane-Et ${ }_{2} \mathrm{O}$, 20:4 $\rightarrow 20: 6)$ furnished $15(0.049 \mathrm{~g}, \mathrm{ca} 3 \%)$ as an oil. ${ }^{1} \mathrm{H}$ NMR (300 $\mathrm{MHz}, \mathrm{CDCl}_{3}$ ): $\delta=7.66-7.35$ (m, two groups of signals, aromatic, $10 \mathrm{H}), 3.86(\mathrm{~m}$ of 6 lines, $J=5.2 \mathrm{~Hz}, 1 \mathrm{H}), 3.71-3.62(\mathrm{~m}, 2 \mathrm{H})$, 3.56 (apparent d, $J=5.5 \mathrm{~Hz}, 2 \mathrm{H}) \mathrm{ppm} .{ }^{13} \mathrm{C} \mathrm{NMR}(75 \mathrm{MHz}$, $\left.\mathrm{CDCl}_{3}\right): \delta=135.7,133.0,130.2,128.1,71.1,65.2,53.4,27.0,19.4$ ppm. HRMS (pos.): calcd. for $\mathrm{C}_{19} \mathrm{H}_{25} \mathrm{KN}_{3} \mathrm{O}_{2} \mathrm{Si}[\mathrm{M}+\mathrm{K}]^{+} 394.1353$; found 394.1347. Method b. Starting from (S)-1,2-isopropylidene glycerol via $(S)$-3-azido-1,2-propane diol 19: The diol 19 (2.55 g, $21.8 \mathrm{mmol})$ in dry DMF $(30 \mathrm{ml})$ and imidazole $(3.2 \mathrm{~g}, 48 \mathrm{mmol})$ was treated with $t \mathrm{BuPh}_{2} \mathrm{SiCl}(6.6 \mathrm{~g}, 6.2 \mathrm{ml}, 14 \mathrm{mmol})$, added dropwise using a syringe during ca 5 min with cooling in ice bath and under argon atmosphere. After addition was complete, the reaction proceeded at r.t. during $6 \mathrm{~h}$. TLC showed a spot $\mathrm{R} f 0.33$ (hexane- $\mathrm{Et}_{2} \mathrm{O}$ 4:1). Extraction $\left(\mathrm{CH}_{2} \mathrm{Cl}_{2}-\mathrm{H}_{2} \mathrm{O}\right)$, drying, evaporation and chromatography in hexane-Et $t_{2} \mathrm{O}$ 3:1 furnished 15 (6.4 g, 82\%) as an oil.

(S)-3-Azido-1,2-propanediol (19): Commercial (S)-2,3isopropylidene glycerol 16 (Fluka) (15,4 g, $116.3 \mathrm{mmol})$ was dissolved in $\mathrm{CH}_{2} \mathrm{Cl}_{2}(150 \mathrm{ml}), \mathrm{Et}_{3} \mathrm{~N}(25 \mathrm{ml})$ and $\mathrm{TsCl}(16.5 \mathrm{~g}, 139$ $\mathrm{mmol})$ were then added. The mixture was stirred for $8 \mathrm{~h}$, isolation after conventional extraction and chromatography in hexaneEtOAc 4:1, yielded 17, $\mathrm{R}_{\mathrm{f}} 0.45,(31.6 \mathrm{~g}, 95 \%), \alpha_{D}-3.6^{\circ}$, c 6, EtOH, lit. ${ }^{[27]}:-4,5^{\circ} \mathrm{c} 1$, EtOH. Compound $17(10.0 \mathrm{~g}, 35 \mathrm{mmol})$ was then reacted with $\mathrm{NaN}_{3}(11.3 \mathrm{~g}, 175 \mathrm{mmol})$ in dry DMF $(120 \mathrm{ml})$ at $65^{\circ}$ $\mathrm{C}$ for $20 \mathrm{~h}$. After extraction and chromatography in hexane- $\mathrm{Et}_{2} \mathrm{O}$ $4: 1, \mathrm{R}_{\mathrm{f}} 0.43$, azide 18 was isolated $(5.3 \mathrm{~g}, 96.5 \%), \alpha_{D}-52.2^{\circ}$ c 5.6 $\mathrm{CHCl}_{3}$, lit. ${ }^{[28]} \alpha_{D}$ not given]. This azide $(5.1 \mathrm{~g}, 32.5 \mathrm{mmol})$ was dissolved in $\mathrm{MeOH}(360 \mathrm{ml})$ and $p$ TSA monohydrate $(0.32 \mathrm{~g}, 1.7$ mmol) was added, the mixture was kept at r.t. during $25 \mathrm{~h} .{ }^{[28]}$ TLC showed more polar product $\mathrm{R}_{\mathrm{f}} 0.40$ hexane-EtOAc 1:3 which chars with a Hanessian's stain. Neutralization with solid $\mathrm{NaHCO}_{3}, 5.3 \mathrm{~g}$ during $45 \mathrm{~min}$., filtration, evaporation and chromatography in hexane-EtOAc 1:3 furnished 19 as an oil (2.8 g, 57\%), $\alpha_{D}+2.8^{\circ}$, c $4.7 \mathrm{CHCl}_{3}, \alpha_{D}-16.2^{\circ}$, c 3.6, MeOH; lit. ${ }^{[29]} \alpha_{D}-10.8^{\circ}$ c $0.98 \mathrm{MeOH}$. ${ }^{1} \mathrm{H}$ NMR (300 MHz, DMSO-d6): $\delta=5.09$ (d, $J=5.1 \mathrm{~Hz}$, exchangeable, $1 \mathrm{H}), 4.67(\mathrm{t}, J=5.6 \mathrm{~Hz}$, exchangeable, $1 \mathrm{H}), 3.68-$ 3.59 ( $\mathrm{m}$ of 15 lines, $1 \mathrm{H}$ ), 3.41-3.14 ( $\mathrm{m}$ of 16 lines, $4 \mathrm{H}$ ) ppm. After $\mathrm{D}_{2} \mathrm{O}$ exchange: $3.66-3.59(\mathrm{~m}$ of 12 lines, $1 \mathrm{H}), 3.36(\mathrm{dd}, J=5.4$, $10.9 \mathrm{~Hz}, 1 \mathrm{H}), 3.28(\mathrm{dd}, J=6.6,10.6 \mathrm{~Hz}, 1 \mathrm{H}), 3.26(\mathrm{dd}, J=3.9$, $12.7 \mathrm{~Hz}, 1 \mathrm{H}), 3.16$ (dd, $J=6.7,12.7 \mathrm{~Hz} 1 \mathrm{H}) \mathrm{ppm} .{ }^{13} \mathrm{C}$ NMR $(75$ MHz, DMSO- $d 6): \delta=74.0,63.2,53.5 \mathrm{ppm}$.

(S)-2-Acetoxy-3-azido-1-tbutyldiphenylsilyloxy propane (20): Conventional acetylation of $\mathbf{1 5}(4.71 \mathrm{~g}, 13.3 \mathrm{mmol})$ in pyridine (30 
$\mathrm{ml})$ and $\mathrm{Ac}_{2} \mathrm{O}(15 \mathrm{ml})$ overnight, followed by extractive work-up, coevaporation with xylenes and exhaustive drying on oil pump furnished 20 as a syrup (5.3 g, quantitatively), $R_{\mathrm{f}} 0.63$ in hexane$\mathrm{Et}_{2} \mathrm{O} 4: 1, \alpha_{D}-14.6^{\circ}, \mathrm{c} 2.8 \mathrm{CHCl}_{3} .{ }^{1} \mathrm{H}$ NMR $\left(300 \mathrm{MHz}, \mathrm{CDCl}_{3}\right): \delta=$ 7.68-7.35 (m, two groups of signals, $10 \mathrm{H}$ ), 5.06 (m of five lines, $J$ $=5.2 \mathrm{~Hz}, 1 \mathrm{H}), 3.79(\mathrm{dd}, J=5.0,10.9 \mathrm{~Hz}, 1 \mathrm{H}), 3.74(\mathrm{dd}, J=5.5$, $10.9 \mathrm{~Hz}, 1 \mathrm{H}$ ), 3.53 (apparent d, $J=5.2 \mathrm{~Hz}, 2 \mathrm{H}), 2.04(\mathrm{~s}, 3 \mathrm{H})$, $1.06(\mathrm{~s}, 9 \mathrm{H}) \mathrm{ppm} .{ }^{13} \mathrm{C} \mathrm{NMR}\left(75 \mathrm{MHz}, \mathrm{CDCl}_{3}\right): \delta=170.3,135.8$, 135.7, 133.1, 130.1, 128.0, 127.9, 72.8, 62.6, 50.9, 26.9, 21.1, 19.4 ppm. HRMS (pos.): calcd. for $\mathrm{C}_{21} \mathrm{H}_{27} \mathrm{~N}_{3} \mathrm{NaO}_{3} \mathrm{Si} \quad[\mathrm{M}+\mathrm{Na}]^{+}$ 420.1714; found 420.1740. Attempted conversion of the azide 20 to get amine 21 via a Staudinger reaction ${ }^{[30]}$ resulted in a complex reaction mixture, which was not investigated.

(S)-3-Amino-1,2-isopropylidenoxy propane (22): This compound was obtained by reduction of the azide $\mathbf{1 8}$ using either a Staudinger reaction $^{[30]}$ or $\mathrm{Na}_{2} \mathrm{~S} 9 \mathrm{H}_{2} \mathrm{O}$ by analogy to the published procedure. ${ }^{[31]}$ Via a Staudinger reaction: To a magnetically stirred solution of $\mathbf{1 8}$ $(1.6 \mathrm{~g}, 10 \mathrm{mmol})$ in THF $(20 \mathrm{ml})$ and water (4 drops) was added $\mathrm{Ph}_{3} \mathrm{P}(3.1 \mathrm{~g}, 12 \mathrm{mmol})$. Evolution of nitrogen started within $c a 30 \mathrm{~s}$ After an overnight reaction the product showed a spot visualized with ninhydrin $\mathrm{R}_{\mathrm{f}} 0.50$ in $\mathrm{CH}_{2} \mathrm{Cl}_{2}-\mathrm{MeOH}-25 \% \mathrm{NH}_{4} \mathrm{OH}$ 20:2:1. Evaporation of volatiles and chromatography in the abovementioned system furnished 22 as yellowish oil (1.06 $\mathrm{g} 70 \%$ ). Via $\mathrm{Na}_{2} \mathrm{~S} \cdot 9 \mathrm{H}_{2} \mathrm{O}$ : To a solution of azide $18(3.35 \mathrm{~g}, 21.3 \mathrm{mmol})$ in $\mathrm{MeOH}(10 \mathrm{ml})$ was added a solution of $\mathrm{Na}_{2} \mathrm{~S} \cdot 9 \mathrm{H}_{2} \mathrm{O}(6.4 \mathrm{~g})$ in $\mathrm{H}_{2} \mathrm{O}$ $(10 \mathrm{ml})$. Stirring was continued for $16 \mathrm{~h}$ at $55^{\circ} \mathrm{C}$. The reaction mixture was transferred to a separatory funnel charged with $\mathrm{Et}_{2} \mathrm{O}$ and $\mathrm{H}_{2} \mathrm{O}$. After exhaustive extraction with $\mathrm{Et}_{2} \mathrm{O}$ (six times), the organic phase was dried and the solvent was evaporated to furnish an yellowish oil. Crude 22 thus obtained was used for the subsequent transformation. $\alpha_{D}+1.1^{\circ}$ c $14, \mathrm{CHCl}_{3}$, lit. $^{[33]} \alpha_{D}+0.9^{\circ} \mathrm{c}$ $1 \mathrm{CHCl}_{3}$, lit. ${ }^{[32]} \alpha_{D}+1.48^{\circ}$ neat. ${ }^{1} \mathrm{H}$ NMR $\left(300 \mathrm{MHz}, \mathrm{CDCl}_{3}\right): \delta=$ 4.13 (ddd, $J=4.3,6.3,12.6 \mathrm{~Hz}, 1 \mathrm{H}), 4.04$ (dd, $J=6.4,7.9 \mathrm{~Hz}, 1$ H), $3.52(\mathrm{dd}, J=6.4,7.9 \mathrm{~Hz}, 1 \mathrm{H}), 2.85(\mathrm{dd}, J=4.4,13.1 \mathrm{~Hz}, 1 \mathrm{H})$, $2.78(\mathrm{dd}, J=7.1,13.1,1 \mathrm{H}), 1.43(\mathrm{~s}, 3 \mathrm{H}), 1.36$ (s, $3 \mathrm{H}), 1.26$ (br s, $2 \mathrm{H}) \mathrm{ppm} .{ }^{13} \mathrm{C} \mathrm{NMR}\left(75 \mathrm{MHz}, \mathrm{CDCl}_{3}\right): \delta=109.2,77.5,67.0,44.8$, 26.9, $25.4 \mathrm{ppm}$.

\section{6-Amino-6-deoxy-1,2;3,4-di-O-isopropylidene-(D)-}

galactopyranose (26): Conventional tosylation of commercial 1,2;3,4-di- $O$-isopropylidene- $(D)$-galactopyranose $23\left(\alpha_{D}-56.8^{\circ}\right.$, c $5.9 \mathrm{CHCl}_{3}$, Acros) in $\mathrm{CH}_{2} \mathrm{Cl}_{2}$, pyridine or $\mathrm{Et}_{3} \mathrm{~N}$ and $\mathrm{TsCl}(1.1 \mathrm{eq})$ overnight, followed by extractive work-up $\left(\mathrm{CH}_{2} \mathrm{Cl}_{2}-\mathrm{H}_{2} \mathrm{O}\right)$ and chromatography in hexane-EtOAc $3: 1, \mathrm{R}_{\mathrm{f}} 0.41$, furnished $24^{[35]}$ (89\%), $\alpha_{D}-62.4^{\circ}$, c $1.7 \mathrm{CHCl}_{3}$, lit. ${ }^{[35]} \alpha_{D}-63^{\circ}, \mathrm{CHCl}_{3}$, c not given. No attempt was made to crystallize this compound. ${ }^{[35]}$ Tosylate 24 was converted to the corresponding 6 -azide 25 by analogy to the procedure for $(L)$-galactose ${ }^{[36]}\left(\mathrm{NaN}_{3} 5 \mathrm{eq}, \mathrm{DMF}, \mathrm{ca} 120^{\circ} \mathrm{C}, 18 \mathrm{~h}\right.$ under argon) to furnish 25 in $91 \%$ yield after extractive work-up $\left(\mathrm{CH}_{2} \mathrm{Cl}_{2}-\mathrm{H}_{2} \mathrm{O}\right)$ and chromatography in hexane-EtOAc 5:1, $\mathrm{R}_{\mathrm{f}} 0.39$; $\alpha_{D}-98^{\circ}$, c $2.4 \mathrm{CHCl}_{3}$. To a magnetically stirred solution of this azide $(3.3 \mathrm{~g}, 11.6 \mathrm{mmol})$ in THF $(50 \mathrm{ml})$ was added $\mathrm{H}_{2} \mathrm{O}(0.3 \mathrm{ml})$ followed by $\mathrm{Ph}_{3} \mathrm{P}(3.7 \mathrm{~g}, 14 \mathrm{mmol})$ by analogy to similar reduction. ${ }^{[30]}$ Evolution of nitrogen started in $c a 30 \mathrm{~s}$. Next day TLC showed that all substrate reacted to form the amine $26 \mathrm{R}_{\mathrm{f}} 0.61$ in $\mathrm{CH}_{2} \mathrm{Cl}_{2}-\mathrm{MeOH}$ 20:3. The solvent was evaporated and the residue was purified by chromatography (gradient) $\mathrm{CH}_{2} \mathrm{Cl}_{2}-\mathrm{MeOH}$ $20: 2 \rightarrow 20: 3$ to furnish 26 as an oil $(2.94 \mathrm{~g}, 98 \%)$. Alternatively, 25 was subjected to catalytic hydrogenation $(10 \% \mathrm{Pd} / \mathrm{C}, \mathrm{EtOH}$, at 50 psi in a Parr apparatus during $6 \mathrm{~h}$ ) to furnish amine $\mathbf{2 6}$ in nearly quantitative yield. $\alpha_{D}-42.1^{\circ}$, c $1.4, \mathrm{MeOH}$; lit. ${ }^{[40]}-53.1^{\circ}$ c $1 \mathrm{CHCl}_{3}$; $\alpha_{D}+48.6^{\circ} \mathrm{c} 1.4 \mathrm{MeOH}$ for the $(L)$-form. ${ }^{[36]}{ }^{1} \mathrm{H}$ NMR $(300 \mathrm{MHz}$, $\left.\mathrm{CDCl}_{3}\right): \delta=5.56(\mathrm{~d}, J=5.0 \mathrm{~Hz}, 1 \mathrm{H}), 4.60(\mathrm{dd}, J=2.3,7.9 \mathrm{~Hz}, 1$ H), $4.32(\mathrm{dd}, J=2.4,5.0 \mathrm{~Hz}, 1 \mathrm{H}), 4.23(\mathrm{dd}, J=1.9,7.9 \mathrm{~Hz}, 1 \mathrm{H})$, 3.84 (ddd, $J=1.7,4.6,7.2 \mathrm{~Hz}, 1 \mathrm{H}$ ), 3.54 (br s, $2 \mathrm{H}$, in the other samples this signal appeared at $2.43 \mathrm{ppm}$ and $2.22 \mathrm{ppm}), 3.04$ (dd, $J=8.0,13.2 \mathrm{~Hz}, 1 \mathrm{H}), 2.96(\mathrm{dd}, J=4.1,13.2 \mathrm{~Hz}, 1 \mathrm{H}), 1.57$ (s, 3 $\mathrm{H}), 1.45$ (s, $3 \mathrm{H}), 1.33$ (s, $6 \mathrm{H}) \mathrm{ppm} .{ }^{13} \mathrm{C} \mathrm{NMR}\left(75 \mathrm{MHz}, \mathrm{CDCl}_{3}\right): \delta$ $=109.5,108.9,96.5,71.8,71.0,70.7,68.3,41.9,26.3,26.2,25.1$, $24.5 \mathrm{ppm}$.

$\begin{array}{lll}\text { 2-Amino-1,3-bis-(tbutyldiphenylsilyloxy) propane } & \text { (31) and } \\ \text { (rac)-2-amino-1-tbutyldiphenylsilyloxy-3-propanol } & \text { (32): } & \text { 2- }\end{array}$ Amino-1,3-propane diol (Acros or Aldrich) (3.85 g, $42.8 \mathrm{mmol}$ ) was solubilized in dry DMF $(36 \mathrm{ml})$ under argon. To this solution was added imidazole (12.6 g, $185.1 \mathrm{mmol})$ followed by $t \mathrm{BuPh}_{2} \mathrm{SiCl}$ (24.7 g, $23.3 \mathrm{ml}, 90.1 \mathrm{mmol})$ using a syringe while maintaining magnetic stirring. After an overnight reaction, TLC showed a UV absorbing compound $\mathrm{R}_{\mathrm{f}} 0.52$ (hexane-EtOAc $2: 1$ ) which chars with ninhydrin upon heating. Extraction $\left(\mathrm{CH}_{2} \mathrm{Cl}_{2}\right.$ - dil. $\left.\mathrm{NaHCO}_{3}\right)$, drying $\left(\mathrm{MgSO}_{4}\right)$, evaporation and chromatography (gradient) hexaneEtOAc $3: 1 \rightarrow 2: 1$, gave 31 as colorless oil (20.3 g, 85\%). Elution with $\mathrm{CH}_{2} \mathrm{Cl}_{2}-\mathrm{MeOH}$ 10:1 furnished monosilylated product $32(0.12$ g, $0.9 \%)$. Data for 31: ${ }^{1} \mathrm{H}$ NMR $\left(300 \mathrm{MHz}, \mathrm{CDCl}_{3}\right): \delta=7.65-7,32$ $(\mathrm{m}$, aromatic, $20 \mathrm{H}), 3.71(\mathrm{dd}, J=5.2,9.8 \mathrm{~Hz}, 2 \mathrm{H}), 3.62(\mathrm{dd}, J=$ $5.8,9.8 \mathrm{~Hz}, 2 \mathrm{H}$ ), 3.00 (m of 5 lines, $J=5.6 \mathrm{~Hz}, 1 \mathrm{H}$ ), 1.51 (s, $2 \mathrm{H}$ ), $1.03(\mathrm{~s}, 18 \mathrm{H}) \mathrm{ppm} .{ }^{13} \mathrm{C} \mathrm{NMR}\left(75 \mathrm{MHz}, \mathrm{CDCl}_{3}\right): \delta=135.8,133.7$, $129.8,127.9,65.9,54.6,27.1,19.5$ ppm. HRMS (pos.): calcd. for $\mathrm{C}_{35} \mathrm{H}_{46} \mathrm{NO}_{2} \mathrm{Si}_{2}[\mathrm{M}+\mathrm{H}]^{+}$568.3061; found 568.3057. Data for 32: ${ }^{1} \mathrm{H}$ NMR (300 MHz, DMSO-d6): $\delta=7.64-7.41(\mathrm{~m}$, aromatic, $10 \mathrm{H})$, 3.59 (dd, $J=6.9,9.7 \mathrm{~Hz}, 1 \mathrm{H}), 3.51(\mathrm{dd}, J=5.8,9.6 \mathrm{~Hz}, 1 \mathrm{H}), 3.45$ (dd, $J=5.3,10.4 \mathrm{~Hz}, 1 \mathrm{H}), 3.33(\mathrm{dd}, J=6.0,10.4 \mathrm{~Hz}, 1 \mathrm{H}), 3.23$ (br s, $2 \mathrm{H}$ ), 2.84 (m of 5 lines, $J=5.7 \mathrm{~Hz}, 1 \mathrm{H}$ ) ppm. ${ }^{13} \mathrm{C}$ NMR $(75$ MHz, DMSO-d6): $\delta=135.1,133.2,129.8,127.9,66.1,63.2,54.3$, 26.7, 18.9 ppm. HRMS (pos.): calcd. for $\mathrm{C}_{19} \mathrm{H}_{28} \mathrm{NO}_{2} \mathrm{Si}[\mathrm{M}+\mathrm{H}]^{+}$ 330.1884; found 330.1894 .

Diethoxyphosphonylmethyl tosylate (33a): Diethylhydroxymethyl phosphonate (Aldrich) $(13.5 \mathrm{~g}, 78 \mathrm{mmol})$ was added to pyridine $(30 \mathrm{ml})$ and $\mathrm{CH}_{2} \mathrm{Cl}_{2}(70 \mathrm{ml})$ and the resulting solution was cooled in an ice-bath under argon atmosphere. Tosyl chloride (14.9 $\mathrm{g}, 78 \mathrm{mmol}$ ) was added and the mixture was allowed to reach $\mathrm{rt}$ during $5 \mathrm{~h}$ and left at $\mathrm{rt}$ for $20 \mathrm{~h}$. The mixture was partitioned between $\mathrm{CH}_{2} \mathrm{Cl}_{2}$ and water. The organic phase was washed with sat. $\mathrm{NaHCO}_{3}$ followed by water, dried, filtered and the volatiles were evaporated. Xylenes were added and evaporated to remove any residual pyridine. Final drying on oil pump yielded crude product $(23.4 \mathrm{~g}, 91 \%)$ which was used without any further purification. The yield obtained was higher than published. ${ }^{[41]}{ }^{1} \mathrm{H}$ NMR $(300 \mathrm{MHz}$, $\left.\mathrm{CDCl}_{3}\right): \delta=7.80(\mathrm{~d}, J=8.3 \mathrm{~Hz}, 2 \mathrm{H}), 7.37(\mathrm{~d}, J=8.0 \mathrm{~Hz}, 2 \mathrm{H})$, 4.23-4.09 (m unresolved, $6 \mathrm{H}), 2.46(\mathrm{~s}, 3 \mathrm{H}), 1.31(\mathrm{t}, J=7.1 \mathrm{~Hz}, 6$ H) ppm. ${ }^{13} \mathrm{C}$ NMR (75 MHz): $\delta=145.6,131.8,130.1,128.3,63.5$ $\left(\mathrm{d}, J_{\mathrm{CP}}=6.4 \mathrm{~Hz}\right), 61.4\left(\mathrm{~d}, J_{\mathrm{CP}}=167.9 \mathrm{~Hz}\right), 21.7,16.4\left(\mathrm{~d}, J_{\mathrm{CP}}=5.7\right.$ $\mathrm{Hz}) \mathrm{ppm} .{ }^{31} \mathrm{P} \mathrm{NMR}\left(121 \mathrm{MHz}, \mathrm{CDCl}_{3}\right): \delta=15.2 \mathrm{ppm}$. Attempts to obtain dithylphosphonomethyloxysulphonoimidazole (to be used instead of triflate 33b or tosylate 33a) by direct reaction of diethylhydroxymethyl phosphonate with sulfuryldiimidazole ${ }^{[44]}$ and $\mathrm{NaH}$, or with sulfuryl chloride and imidazole ${ }^{[43]}$, followed by addition of the amine 39 e.g. furnished no traces of the expected product $\mathbf{4 0}$ (see below).

Diethoxyphosphonylmethyl triflate (33b): Compound 33b was prepared from diethoxyhydroxymethylphosphonate, trifluoromethylsulfonyl anhydride and 2,6-lutidine as published ${ }^{[42]}$ (an alternative is to use $\mathrm{CF}_{3} \mathrm{SO}_{2} \mathrm{Cl}$ and $\mathrm{NaH}^{[47]}$ ) and was used as a crude product without any further purification (such as distillation ${ }^{[42]}$ ). This material was most probably contaminated with bis(diethylphosphonomethyl) ether. ${ }^{[47]}$ Diethylhydroxymethyl phosphonate $(5.2 \mathrm{~g}, 4.5 \mathrm{ml}, 30.6 \mathrm{mmol})$ in dry $\mathrm{CH}_{2} \mathrm{Cl}_{2}(50 \mathrm{ml}$, freshly distilled from $\mathrm{CaH}_{2}$ ) and 2,6-lutidine (dried by storage over $\mathrm{KOH}, 4.3 \mathrm{ml}, 37.6 \mathrm{mmol}$ ) was cooled to $c a-50{ }^{\circ} \mathrm{C}$ (external temp.) 
under argon. To this solution was slowly added trifluoromethylsulfonic anhydride $(10.1 \mathrm{~g}, 6.0 \mathrm{ml}, 35.6 \mathrm{mmol})$ via a syringe during $c a 10 \mathrm{~min}$ while maintaining magnetic stirring. After addition was complete, the temperature was increased slowly to $\mathrm{ca} 10^{\circ} \mathrm{C}$ during $90 \mathrm{~min}$, whereupon extraction was performed $\left(\mathrm{CH}_{2} \mathrm{Cl}_{2}\right.$-dil. $\left.\mathrm{HCl}\right)$. The organic phase was washed once with icecold $\mathrm{H}_{2} \mathrm{O}$, dried $\left(\mathrm{MgSO}_{4}\right)$, filtered, evaporated and finally dried on oil pump to furnish $9.5 \mathrm{~g}$ of red oil (theoretical yield is $9.2 \mathrm{~g}$ ). This material was used as such immediately after preparation.

\section{(S)-3-N-Diethylphosphonomethyl-1,2-isopropylidenoxypropyl} amine (34): $(S)$-Amine $22(1.06 \mathrm{~g}, 8.0 \mathrm{mmol})$, tosylate 33a (3.7 g, $11.5 \mathrm{mmol})$ and powdered molecular sieves $4 \AA$ ( $2 \mathrm{~g})$ in dry DMF $(25 \mathrm{ml})$ were stirred under argon at $80{ }^{\circ} \mathrm{C}$ during $20 \mathrm{~h}$. TLC showed the product $34 \mathrm{R}_{\mathrm{f}} 0.28\left(\mathrm{CH}_{2} \mathrm{Cl}_{2}-\mathrm{MeOH} 20: 1\right)$ revealed with ninhydrin. Unreacted amine remained at the application point. The solid material was filtered off, and extraction was performed $\left(\mathrm{CH}_{2} \mathrm{Cl}_{2}-\mathrm{H}_{2} \mathrm{O}\right)$. The organic phase was dried $\left(\mathrm{MgSO}_{4}\right)$, and the solvent was evaporated. Chromatography in $\mathrm{CH}_{2} \mathrm{Cl}_{2}-\mathrm{MeOH} 10: 1$ furnished $34(0.49 \mathrm{~g}, 21.5 \%)$ as colorless oil. For the NMR data see compound 40. Prolongation of the reaction time and/or using of higher temperature did not improve the yield. $\alpha_{D}+2.4^{\circ}$, c 3.7, $\mathrm{CHCl}_{3}$; HRMS (pos.): calcd. for $\mathrm{C}_{11} \mathrm{H}_{25} \mathrm{NO}_{5} \mathrm{P}[\mathrm{M}+\mathrm{H}]^{+}$282.1465; found 282.1478 .

\section{6-Deoxy-6- $N$-diethylphosphonomethyl-1,2;3,4-di- $O$ -}

isopropylidene-(D)-galactosamine (35): Tosylate $33 \mathbf{a}(0.88 \mathrm{~g}$, $2.78 \mathrm{mmol})$ and galactosylamine $26(0.72 \mathrm{~g}, 2.78 \mathrm{mmol})$ in dry DMF $(10 \mathrm{ml})$ and powdered mol. sieves $4 \AA(3 \mathrm{~g})$ were stirred under argon at $80^{\circ} \mathrm{C}$ for $20 \mathrm{~h}$. TLC showed a new product $\mathrm{R}_{\mathrm{f}} 0.40$ $\left(\mathrm{CH}_{2} \mathrm{Cl}_{2}-\mathrm{MeOH}\right.$ 20:0.8). Unreacted 33a and 26 were still present. The solid material was filtered out using a sintered glass, and the filtrate was extracted $\left(\mathrm{CH}_{2} \mathrm{Cl}_{2}-\mathrm{H}_{2} \mathrm{O}\right)$. After usual work-up the residue was purified by chromatography in $\mathrm{CH}_{2} \mathrm{Cl}_{2}-\mathrm{MeOH}$ 20:0.8 to furnish 35 as an oil $(0.42 \mathrm{~g}, 37 \%)$. ${ }^{1} \mathrm{H}$ NMR $\left(300 \mathrm{MHz}, \mathrm{CDCl}_{3}\right)$ : $\delta=5.52(\mathrm{~d}, J=5.0 \mathrm{~Hz}, 1 \mathrm{H}), 4.59(\mathrm{dd}, J=2.3,7.9 \mathrm{~Hz}, 1 \mathrm{H}), 4.31$ $(\mathrm{dd}, J=2.4,5.1 \mathrm{~Hz}, 1 \mathrm{H}), 4.20-4.10(\mathrm{~m}, 5 \mathrm{H}), 3.89$ (ddd, $J=1.7$, 4.4, 8.1 Hz, $1 \mathrm{H}), 3.03(\mathrm{dd}, J=2.6,12.5 \mathrm{~Hz}, 2 \mathrm{H}), 2.97(\mathrm{dd}, J=4.3$ $7.6 \mathrm{~Hz}, 1 \mathrm{H}), 2.85(\mathrm{dd}, J=4.4,12.7 \mathrm{~Hz}, 1 \mathrm{H}), 1.94(\mathrm{br} \mathrm{s}$, exchangeable, $1 \mathrm{H}), 1.52(\mathrm{~s}, 3 \mathrm{H}), 1.44(\mathrm{~s}, 3 \mathrm{H}), 1.36(\mathrm{~s}, 3 \mathrm{H}), 1.34$ $(\mathrm{s}, 3 \mathrm{H}), 1.33(\mathrm{~s}, 3 \mathrm{H}), 1.31(\mathrm{~s}, 3 \mathrm{H}) \mathrm{ppm} .{ }^{13} \mathrm{C} \mathrm{NMR}(75 \mathrm{MHz}$, $\left.\mathrm{CDCl}_{3}\right): \delta=109.4,108.6,96.5,72.0,71.0,70.7,66.7,62.2\left(\mathrm{~d}, J_{\mathrm{CP}}\right.$ $=6.6 \mathrm{~Hz}), 50.8\left(\mathrm{~d}, J_{\mathrm{CP}}=14.9 \mathrm{~Hz}\right), 45.1\left(\mathrm{~d}, J_{\mathrm{CP}}=155.4 \mathrm{~Hz}\right), 26.2$, 26.1, 25.1, 24.5, $16.6\left(\mathrm{~d}, J_{\mathrm{CP}}=5.7 \mathrm{~Hz}\right) \mathrm{ppm} .{ }^{31} \mathrm{P} \mathrm{NMR}(121 \mathrm{MHz}$, $\mathrm{CDCl}_{3}$ ): $\delta=25.9$ ppm. HRMS (pos.): calcd. for $\mathrm{C}_{17} \mathrm{H}_{33} \mathrm{NO}_{8} \mathrm{P}$ $[\mathrm{M}+\mathrm{H}]^{+}$410.1938; found 410.1935.

\section{(Rac)-3-N-Diethylphosphonomethyl-1,2-isopropylidenoxy-}

propyl amine (40): Tosylphosphonate 33a $(3.7 \mathrm{~g}, 7.6 \mathrm{mmol})$ was added to a solution of (rac)-3-amino-1,2-isopropylidenoxy propane 39 (Aldrich) $(1.0 \mathrm{~g}, 7.6 \mathrm{mmol})$ in dry DMF $(12 \mathrm{ml})$ followed by powdered $4 \AA$ molecular sieves $(2 \mathrm{~g})$. The mixture was stirred under argon during $12 \mathrm{~h}$ at $80^{\circ} \mathrm{C}$. TLC showed the product $40 \mathrm{R}_{\mathrm{f}}$ $0.28\left(\mathrm{CH}_{2} \mathrm{Cl}_{2}-\mathrm{MeOH} 20: 1\right)$, revealed with ninhydrin. Unreacted amine remained at the application point. The solid material was filtered off, and extraction was performed $\left(\mathrm{CH}_{2} \mathrm{Cl}_{2}-\mathrm{H}_{2} \mathrm{O}\right)$. The organic phase was dried $\left(\mathrm{MgSO}_{4}\right)$, and the solvent was evaporated. Chromatography in $\mathrm{CH}_{2} \mathrm{Cl}_{2}-\mathrm{MeOH}$ 10:1 furnished $\mathbf{4 0}$ as yellowish oil $(0.86 \mathrm{~g}, 30.2 \%)$. Prolongation of the reaction time and/or using of higher temperature did not improve the yield. ${ }^{1} \mathrm{H}$ NMR $(300$ MHz, DMSO- $d 6$ ): $\delta=4.12$ (m of 5 lines, $J=6.0 \mathrm{~Hz}, 1 \mathrm{H}), 4.02(\mathrm{~m}$ of 5 lines, $J=7.3 \mathrm{~Hz}, 4 \mathrm{H}), 3.96(\mathrm{dd}, J=6.5,8.0 \mathrm{~Hz}, 1 \mathrm{H}), 3.60$ (dd, $J=6.5,8.0 \mathrm{~Hz}, 1 \mathrm{H}), 3.31$ (br s, exchangeable, $1 \mathrm{H}), 2.94$ (d, $J$ $=11.4 \mathrm{~Hz}, 2 \mathrm{H}), 2.71(\mathrm{~d}, J=2.1 \mathrm{~Hz}, 1 \mathrm{H}), 2.69(\mathrm{~d}, J=1.7 \mathrm{~Hz}, 1 \mathrm{H})$, $1.31(\mathrm{~s}, 3 \mathrm{H}), 1.26(\mathrm{~s}, 3 \mathrm{H}), 1.23(\mathrm{t}, J=7.1 \mathrm{~Hz}, 6 \mathrm{H}) \mathrm{ppm} .{ }^{13} \mathrm{C} \mathrm{NMR}$ $(75 \mathrm{MHz}, \mathrm{DMSO}-d 6): \delta=108.1,74.8,66.9,61.2\left(\mathrm{~d}, J_{\mathrm{CP}}=6.5 \mathrm{~Hz}\right)$, $52.7\left(\mathrm{~d}, J_{\mathrm{CP}}=12.8 \mathrm{~Hz}\right), 44.3\left(\mathrm{~d}, J_{\mathrm{CP}}=154.0 \mathrm{~Hz}\right), 26.7,25.3,16.3$ $\left(\mathrm{d}, J_{\mathrm{CP}}=5.5 \mathrm{~Hz}\right) \mathrm{ppm} .{ }^{31} \mathrm{P}$ NMR $(121 \mathrm{MHz}, \mathrm{DMSO}-d \sigma): \delta=26.1$ ppm and impurity (ca 6\%) at 24.4 ppm. HRMS (pos.): calcd. for $\mathrm{C}_{11} \mathrm{H}_{25} \mathrm{NO} 5 \mathrm{P}[\mathrm{M}+\mathrm{H}]^{+}$282.1465; found 282.1462 .

1,3-Di-tbutyldiphenylsilyloxy-2-( $N$-diethoxyphosphonomethyl)propyl amine (36): Triflate $33 \mathrm{~b}(4.2 \mathrm{~g}, \mathrm{ca} 14 \mathrm{mmol})$ was added to a solution of the amine $31(7.9 \mathrm{~g}, 14 \mathrm{mmol})$ in dry THF $(30 \mathrm{ml})$ under argon. The reaction mixture became blue. Some warming took place. After $8 \mathrm{~h}$ at r.t., the newly formed compound 36 showed $\mathrm{R}_{\mathrm{f}} 0.31$ (hexane-EtOAc 3:2). Extractive work-up $\left(\mathrm{CH}_{2} \mathrm{Cl}_{2}-\right.$ $\mathrm{H}_{2} \mathrm{O}$ ) and chromatography in hexane-EtOAc 3:2 furnished the product (5.65 g, 56.6\%). When tosylate 33a was used instead of 33b (in DMF at $100^{\circ}$, overnight), 36 was isolated in $14 \%$ yield. ${ }^{1} \mathrm{H}$ NMR (300 MHz, $\left.\mathrm{CDCl}_{3}\right): \delta=7.64-7.32(\mathrm{~m}$, aromatic, $20 \mathrm{H}), 4.10$ (m of 5 lines, $J=7.3 \mathrm{~Hz}, 4 \mathrm{H}$ ), 3.69 (apparent d, $J=5.7 \mathrm{~Hz}, 4 \mathrm{H}$ ), $2.91\left(\mathrm{~d}, J_{\mathrm{HP}}=13.5 \mathrm{~Hz}, 2 \mathrm{H}\right), 2.80(\mathrm{~m}$ of 5 lines, $J=5.6 \mathrm{~Hz}, 1 \mathrm{H})$, 1.86 (br s, $1 \mathrm{H}), 1.28$ (t, $J=7.1 \mathrm{~Hz}, 6 \mathrm{H}), 1.02$ (s, 18H) ppm. ${ }^{13} \mathrm{C}$ NMR (75 MHz, $\left.\mathrm{CDCl}_{3}\right): \delta=135.7,133.6,129.9,127.9,63.4,62.4$, $62.2\left(\mathrm{~d}, J_{\mathrm{CP}}=6.8 \mathrm{~Hz}\right), 43.7\left(\mathrm{~d}, J_{\mathrm{CP}}=156.3 \mathrm{~Hz}\right), 27.0,19.4,16.6(\mathrm{~d}$, $\left.J_{\mathrm{CP}}=5.6 \mathrm{~Hz}\right)$ ppm. ${ }^{31} \mathrm{P}$ NMR $\left(121 \mathrm{MHz}, \mathrm{CDCl}_{3}\right): \delta=26.0 \mathrm{ppm}$. HRMS (pos.): calcd. for $\mathrm{C}_{40} \mathrm{H}_{57} \mathrm{NO}_{5} \mathrm{PSi}_{2}[\mathrm{M}+\mathrm{H}]^{+} 718.3507$; found 718.3517 .

\section{(Rac)-3- $N$-(diethylphosphonomethyl)amino-1-triphenyl- methyloxy-2-propanol (37) and (rac)-3- $N$-bis- (diethylphosphonomethyl)amino-1-O-triphenylmethyloxy-2-}

propanol (38): Via triflate 33b: The triflate 33b (0.5 g, ca 1.6 mmol) was added to a solution of 6 in THF $(15 \mathrm{ml})$ under argon atmosphere. The mixture warmed up and became blue. After $8 \mathrm{~h}$ at r.t., TLC showed the bis-phosphonate $\mathbf{3 8} \quad \mathrm{R}_{\mathrm{f}} \quad 0.55$ and monophosphonate $37 \mathrm{R}_{\mathrm{f}} 0.47\left(\mathrm{CH}_{2} \mathrm{Cl}_{2}-\mathrm{MeOH} 20: 1.3\right)$. Extractive work-up $\left(\mathrm{CH}_{2} \mathrm{Cl}_{2}-\mathrm{H}_{2} \mathrm{O}\right)$ followed by chromatography in $\mathrm{CH}_{2} \mathrm{Cl}_{2}-$ $\mathrm{MeOH} 20: 1.1$ furnished $38(0.23 \mathrm{~g}, 22 \%)$ and $37(0.14 \mathrm{~g}, 18 \%)$. Via tosylate 33a: To a DMF $(10 \mathrm{ml})$ solution of the amine $6(0.81 \mathrm{~g}$, $2.4 \mathrm{mmol})$ was added tosylate 33a $(0.84 \mathrm{~g}, 2.6 \mathrm{mmol})$ and this mixture was incubated at $100{ }^{\circ} \mathrm{C}$ under argon during $9 \mathrm{~h}$. TLC profile was similar to that described above, but additionally minor byproducts were present, which made the purification difficult. Extractive work-up and chromatography furnished 37 as a syrup (0.34 g, 27\%). The doubly phosphonylated compound 38 was not isolated. Data for 38: ${ }^{1} \mathrm{H}$ NMR (300 MHz, $\left.\mathrm{CDCl}_{3}\right)$ : $\delta=7.45-7.18$ (m, two groups of signals, $15 \mathrm{H}$ ), 4.11 (m of 6 lines, $J=7.4 \mathrm{~Hz}, 8$ $\mathrm{H}), 3.87-3.80(\mathrm{~m}$ of 9 lines, $1 \mathrm{H}), 3.30-3.15(\mathrm{~m}, 5 \mathrm{H}), 3.09-3.03(\mathrm{~m}$, $2 \mathrm{H}), 5.89(\mathrm{dd}, J=8.8,13.5 \mathrm{~Hz}, 1 \mathrm{H}), 1.30(\mathrm{dt}, J=3.7,7.0 \mathrm{~Hz}, 12$ H) ppm. ${ }^{13} \mathrm{C}$ NMR $\left(75 \mathrm{MHz}, \mathrm{CDCl}_{3}\right): \delta=144.1,128.8,127.9$, $127.1,86.7,68.8,65.7,62.3,62.2,62.2,62.1,62.1,61.1,61.1,61.0$, $52.1\left(\mathrm{~d}, J_{\mathrm{CP}}=154.6 \mathrm{~Hz}\right), 16.7,16.6,16.6 \mathrm{ppm} .{ }^{31} \mathrm{P} \mathrm{NMR}(121 \mathrm{MHz}$, $\mathrm{CDCl}_{3}$ ): $\delta=25.5$ ppm. HRMS (pos.): calcd. for $\mathrm{C}_{32} \mathrm{H}_{45} \mathrm{NNaO}_{8} \mathrm{P}_{2}$ $[\mathrm{M}+\mathrm{Na}]^{+}$656.2513; found: 656.2504. Data for 37: ${ }^{1} \mathrm{H}$ NMR $(300$ $\mathrm{MHz}, \mathrm{CDCl}_{3}$ ): $\delta=7.44-7.19$ (m, two groups of signals, $15 \mathrm{H}$ ), 4.10 (m of 5 lines, $J=7.3 \mathrm{~Hz}, 4 \mathrm{H}$ ), 3.86 (unresolved $\mathrm{m}$ of 5 lines, $1 \mathrm{H}$ ), $3.17(\mathrm{dd}, J=5.8,9.4 \mathrm{~Hz}, 1 \mathrm{H}), 3.12(\mathrm{dd}, J=5.1,9.5 \mathrm{~Hz}, 1 \mathrm{H}), 2.97$ (apparent dd, $J=2.5,12.3 \mathrm{~Hz}, 2 \mathrm{H}), 2.90-2.70$ (m, $3 \mathrm{H}), 1.29$ (t, $J$ $=7.1 \mathrm{~Hz}, 6 \mathrm{H}) \mathrm{ppm} .{ }^{13} \mathrm{C} \mathrm{NMR}\left(75 \mathrm{MHz}, \mathrm{CDCl}_{3}\right): \delta=144.0,128.8$, $128.0,127.2,86.8,69.3,66.1,62.2\left(\mathrm{~d}, J_{\mathrm{CP}}=6.6 \mathrm{~Hz}\right), 53.8\left(\mathrm{~d}, J_{\mathrm{CP}}=\right.$ $13.6 \mathrm{~Hz}), 45.3\left(\mathrm{~d}, J_{\mathrm{CP}}=153.3 \mathrm{~Hz}\right), 16.6\left(\mathrm{~d}, J_{\mathrm{CP}}=6.0 \mathrm{~Hz}\right) \mathrm{ppm} .{ }^{31} \mathrm{P}$ NMR (121 MHz, $\left.\mathrm{CDCl}_{3}\right): \delta=26.0 \mathrm{ppm}$. HRMS (pos.): calcd. for $\mathrm{C}_{27} \mathrm{H}_{35} \mathrm{NO}_{5} \mathrm{P}[\mathrm{M}+\mathrm{H}]^{+}$484.2247; found 484.2247.

Thymin-1-yl acetic acid (41): Compound 41 was obtained by direct alkylation of thymine with chloroacetic acid in alkaline medium in $46 \%$ yield, as described. ${ }^{[45]}{ }^{1} \mathrm{H}$ NMR $(300 \mathrm{MHz}$, DMSO-d6): $\delta=13.1$ (br s, exchangeable, $1 \mathrm{H}$ ), 7.49 (unresolved q, $J=1.1 \mathrm{~Hz}, 1 \mathrm{H}), 4.37(\mathrm{~s}, 2 \mathrm{H}), 1.75(\mathrm{~d}, J=1.1 \mathrm{~Hz}, 3 \mathrm{H}) \mathrm{ppm} .{ }^{13} \mathrm{C}$ 
NMR (75 MHz): $\delta=169.7,164.34,151.0,141.8,108.4,48.4,11.9$ ppm.

\section{(S)-3'-N-(Diethylphosphonomethyl)-1',2'-isopropylidenoxy-} propyl-thymin-1-yl acetamide (42): Coupling procedure as described below was performed using the amine $34(0.42 \mathrm{~g}, 3.2$ mmol), thymine acetic acid 41 (1.8 g, $9.6 \mathrm{mmol})$, DCC (2.0 g, 9.6 $\mathrm{mmol})$ and HBT $(1.0 \mathrm{~g}, 7.0 \mathrm{mmol})$ in dry DMF $(20 \mathrm{ml})$ during $24 \mathrm{~h}$ Work-up and chromatography yielded $42(0.55 \mathrm{~g}, 82 \%) . \alpha_{D}-18.2^{\circ}$, c 3.1, $\mathrm{CHCl}_{3}$. HRMS (pos.): calcd. for $\mathrm{C}_{18} \mathrm{H}_{31} \mathrm{~N}_{3} \mathrm{O}_{8} \mathrm{P}[\mathrm{M}+\mathrm{H}]^{+}$ 448.1843; found: 448.1846 .

\section{((6' $-N$-6 $6^{\prime}$-Deoxy-1',2'; $3^{\prime}, 4^{\prime}$-di-O-isopropylidene-(D)-} galactopyranosyl))- $N$-(diethylphosphonomethyl)-thymin-1-yl acetamide (43): To a magnetically stirred solution of the amine 35 $(0.41 \mathrm{~g}, 1 \mathrm{mmol})$ and thymine acetic acid $41(0.368 \mathrm{~g}, 2 \mathrm{mmol})$ in dry DMF $(10 \mathrm{ml})$ was added dicyclohexylcarbodiimide DCC $(0.412 \mathrm{~g}, 2 \mathrm{mmol})$ and hydroxybenzotriazole HBT $(0.27 \mathrm{~g}, 2$ mmol) under argon. The mixture became turbid in few seconds. After $36 \mathrm{~h}$ of stirring the solids were filtered off using a sintered glass. The filtrate was extracted using $\mathrm{CH}_{2} \mathrm{Cl}_{2}$ and aq $\mathrm{NaHCO}_{3}$. The organic phase was dried $\left(\mathrm{MgSO}_{4}\right)$ and the volatiles were evaporated. The product which has $\mathrm{R}_{\mathrm{f}} 0.38$ in $\mathrm{CH}_{2} \mathrm{Cl}_{2}-\mathrm{MeOH}$ 20:1.4 was isolated by chromatography in the same system to furnish $43(0.52 \mathrm{~g}, 92 \%)$. $\alpha_{D}-29.3^{\circ} \mathrm{c} 4.1 \mathrm{CHCl}_{3}$. The NMR spectra of 43 (just like of 43,44 and 45) are very complex due to the couplings to ${ }^{31} \mathrm{P}$ and the presence of syn/anti isomers around the amide bond. ${ }^{1} \mathrm{H} \mathrm{NMR}\left(500 \mathrm{MHz}, \mathrm{CDCl}_{3}\right): \delta=9.42$ (major) and 9.36 (minor) ( $2 \mathrm{x}$ br s, $c a$ 6:4 proportion rotamers, exchangeable, 1 $\mathrm{H}), 7.01$ (minor) and 6.93 (major) $(2 \mathrm{x} \mathrm{d}$, rotamers, $J=1.1 \mathrm{~Hz}, 1$ $\mathrm{H}), 5.53$ (major) and 5.47 (minor) $(2 \mathrm{x} \mathrm{d}$, rotamers, $J=4.9 \mathrm{~Hz}, 1$ $\mathrm{H}), 4.95$ (major) and 4.86 (minor) $(2 \mathrm{x} \mathrm{d}, J=16.6 \mathrm{~Hz}, 1 \mathrm{H}), 4.68$ (d, $J=16.5 \mathrm{~Hz})$ and $4.65(\mathrm{dd}, J=2.2,8.0 \mathrm{~Hz})(1 \mathrm{H}), 4.57(\mathrm{dd}, J=2.2$, $7.9 \mathrm{~Hz})$ and $4.52(\mathrm{~d}, J=16.5 \mathrm{~Hz})(1 \mathrm{H}), 4.40(\mathrm{t}, J=15.1 \mathrm{~Hz}, 0.5 \mathrm{H})$, 4.35 (major) and 4.28 (minor) $(2 \mathrm{x} \mathrm{dd}$, rotamers, $J=2.3,4.9 \mathrm{~Hz}, 1$ $\mathrm{H}$ ), 4.23-3.99 (unresolved $\mathrm{m}, 6.5 \mathrm{H}$ ), 3.94-3.83 (unresolved m, 1.5 $\mathrm{H}), 3.71(\mathrm{dd}, J=3.2,15.7 \mathrm{~Hz}, 0.5 \mathrm{H}), 3.46-3.36(\mathrm{~m}, 1 \mathrm{H}), 1.91$ (major) and 1.89 (minor) $(2 \mathrm{x} \mathrm{s}$, rotamers, $3 \mathrm{H}), 1.52-1.26(\mathrm{~m}, 18$ H) ppm. ${ }^{13} \mathrm{C}$ NMR $\left(75 \mathrm{MHz}, \mathrm{CDCl}_{3}\right): \delta=167.7,167.6,167.6$, 164.5, 151.2 (major) and 151.1 (minor), 141.3 (minor) and 141.2 (major), 110.6 (major) and 110.5 (minor), 109.7 (major) and 109.6 (minor), 109.1 (minor) and 109.0 (major), 96.4 (major) and 96.3 (minor), 71.6 (minor) and 71.4 (major), 70.9, 70.5, 64.7, 63.1, 63.0, 62.97, 62.93, 62.8, 62.7, 62.6, 48.8, 48.3, 48.1, 47.5, 45.1 (minor, d, $J_{\mathrm{CP}}=156.9 \mathrm{~Hz}$ ), 40.4 (major, d, $J_{\mathrm{CP}}=155.8 \mathrm{~Hz}$ ), 26.2, 26.1, 26.0, $25.1,24.9,24.5,24.4,16.7,16.6,16.5,16.4,16.3,12.5$ (major) and 12.4 (minor) ppm. ${ }^{31} \mathrm{P}$ NMR (121 MHz, $\mathrm{CDCl}_{3}$ ): $\delta=21.44$ (major) and 21.13 (minor) ppm. HRMS (pos.): calcd. for $\mathrm{C}_{24} \mathrm{H}_{38} \mathrm{~N}_{3} \mathrm{NaO}_{11} \mathrm{P}$ $[\mathrm{M}+\mathrm{Na}]^{+}$598.2136; found 598.2143.

\section{2- $N$-(Diethylphosphonomethyl)-1,3-bis-tbutyldiphenylsilyloxy-} propyl-thymin-1-yl acetamide (44): The amine 36 (3.9 g, 5.4 $\mathrm{mmol})$ and thymine-1-yl-acetic acid $41(3.0 \mathrm{~g}, 16.2 \mathrm{mmol})$ were condensed using DCC (3.3 g, $16.2 \mathrm{mmol})$ and HBT (1.4 g, 10 $\mathrm{mmol})$ in dry DMF $(60 \mathrm{ml})$ during $48 \mathrm{~h}$ under argon as described above. TLC showed the product $\mathbf{4 4} \mathrm{R}_{\mathrm{f}} 0.32$ (hexane-EtOAc 1:4). After usual work-up and chromatography in hexane-EtOAc 1:4, $1.16 \mathrm{~g}, 24 \%$ of 44 was obtained. ${ }^{1} \mathrm{H}$ NMR $\left(300 \mathrm{MHz}, \mathrm{CDCl}_{3}\right): \delta=$ 8.97 (minor) and 8.88 (major) (1:2 proportion, $2 \mathrm{x}$ br s, rotamers, exchangeable, $1 \mathrm{H}), 7.59-7.34(\mathrm{~m}$, aromatic, $20 \mathrm{H}), 6.89$ (minor) and 6.50 (major) $(2 \mathrm{x}$ unresolved q, rotamers, $J c a 1 \mathrm{~Hz}, 1 \mathrm{H}), 4.68$ (minor) and 4.29 (major) (2 x br s, rotamers, $2 \mathrm{H}), 4.15-3.69$ (unresolved $\mathrm{m}, 11 \mathrm{H}$ ), 1.88 (minor) and 1.79 (major) $(2 \mathrm{x}$ unresolved d, $J c a 1 \mathrm{~Hz}, 3 \mathrm{H}$ ), 1.23 (minor) and 1.16 (major) $(2 \mathrm{x} \mathrm{t}$, rotamers, $J=7.1 \mathrm{~Hz}, 6 \mathrm{H}), 1.03$ (major) and 1.00 (minor) $(2 \mathrm{x} \mathrm{s}$, rotamers, $18 \mathrm{H}) \mathrm{ppm} .{ }^{13} \mathrm{C} \mathrm{NMR}\left(75 \mathrm{MHz}, \mathrm{CDCl}_{3}\right): \delta=167.7$,
164.5, 151.1 (minor) and 150.9 (major), 141.2 (minor) and 141.1 (major), 135.7, 135.6, 133.2, 133.1, 132.9, 132.8, 130.2, 130.1, 129.9, 128.1, 127.9, 110.5 (minor) and 110.3 (major), 63.0 (minor, $\mathrm{d}, J_{\mathrm{CP}}=7.4 \mathrm{~Hz}$ ), 62.5 (major, d, $J_{\mathrm{CP}}=6.5 \mathrm{~Hz}$ ), 62.4 (minor) and 62.2 (major), 60.2, 48.6 (minor) and 48.2 (major), 39.1 (d, $J_{\mathrm{CP}}=$ 158.7 Hz), 27.0 (major) and 26.9 (minor), 19.3 (major) and 19.2 (minor), 16.6 (minor, d, $J_{\mathrm{CP}}=5.3 \mathrm{~Hz}$ ), 16.4 (major, d, $J_{\mathrm{CP}}=6.0$ $\mathrm{Hz}$ ), 12.4 ppm. ${ }^{31} \mathrm{P}$ NMR (121 MHz, $\left.\mathrm{CDCl}_{3}\right): \delta=22.47$ (major) and 20.89 (minor) ppm. HRMS (pos.): calcd. for $\mathrm{C}_{47} \mathrm{H}_{62} \mathrm{~N}_{3} \mathrm{NaO}_{8} \mathrm{PSi}_{2}[\mathrm{M}+\mathrm{Na}]^{+}$906.3705; found: 906.3716 .

\section{(Rac)-3- $N$-(Diethylphosphonomethyl)-1,2-O-}

isopropylidenoxypropyl-thymin-1-yl acetamide (45): To a magnetically stirred solution of the amine $\mathbf{4 0}(0.3 \mathrm{~g}, 1.1 \mathrm{mmol})$ and thymin-1-yl-acetic acid $\mathbf{4 1}^{[45]}(0.55 \mathrm{~g}, 3.0 \mathrm{mmol})$ in dry DMF (10 $\mathrm{ml}$ ), was added dicyclohexylcarbodiimide (DCC, $0.62 \mathrm{~g}, 3.0 \mathrm{mmol}$ ) and 1-hydroxybenzotriazole (HBT, $0.27 \mathrm{~g}, 2.0 \mathrm{mmol}$ ) under argon. The mixture became turbid in few seconds. Stirring was continued overnight. TLC showed the product $\mathrm{R}_{\mathrm{f}} 0.38\left(\mathrm{CH}_{2} \mathrm{Cl}_{2}-\mathrm{MeOH}\right.$ 20:1.3). The solids were filtered off using a sintered glass. The filtrate was transferred to a separatory funnel charged with $\mathrm{CH}_{2} \mathrm{Cl}_{2}$ and aq $\mathrm{NaHCO}_{3}$ and extraction was performed. The organic phase was dried $\left(\mathrm{MgSO}_{4}\right)$ and volatiles were evaporated. Chromatography of the residue (gradient $\mathrm{CH}_{2} \mathrm{Cl}_{2}-\mathrm{MeOH}$ $20: 1.2 \rightarrow 20: 1.4)$ furnished 45 as a foam $(0.43 \mathrm{~g}, 90.1 \%) .{ }^{1} \mathrm{H}$ NMR (300 MHz, $\left.\mathrm{CDCl}_{3}\right): \delta=9.86$ (br s, exchangeable, $1 \mathrm{H}$ ), 7.04 (major) and 7.00 (minor) $(2 \times \mathrm{br} \mathrm{s}$, rotamers, proportion $3: 2,1 \mathrm{H})$, 4.88-3.67 (unresolved $\mathrm{m}, 13 \mathrm{H}), 1.90$ (s, $3 \mathrm{H}), 1.47-1.27$ (unresolved $\mathrm{m}, 12 \mathrm{H}$ ) ppm. ${ }^{13} \mathrm{C} \mathrm{NMR}\left(75 \mathrm{MHz}, \mathrm{CDCl}_{3}\right): \delta=167.7$ (major) and 167.3 (major), 164.5 (major) and 164.4 (minor), 151.4 (major) and 151.3 (minor), 141.2 (major) and 141.1 (minor), 110.8 (major) and 110.5 (minor), 109.7, 75.4 (major) and 73.8 (minor), 67.1 (major) and 67.0 (minor), 63.2, 63.1, 63.0, 62.9, 62.9, 62.8, 62.7, 50.8 (minor) and 49.7 (major), 48.4 (major) and 48.1 (minor), 44.7 (major, d, $J_{\mathrm{CP}}=157.2 \mathrm{~Hz}$ ), 41.5 (minor, d, $J_{\mathrm{CP}}=155.1 \mathrm{~Hz}$ ), 26.8, 25.5 (major) and 25.3 (minor), 16.7 (major, d, $J_{\mathrm{CP}}=5.4 \mathrm{~Hz}$ ), 16.6 (minor, d, $J_{\mathrm{CP}}=5.6 \mathrm{~Hz}$ ), 12.5 (minor) and 12.4 (major) ppm. ${ }^{31} \mathrm{P}$ NMR (121 MHz, $\mathrm{CDCl}_{3}$ ): $\delta=21.40$ (minor) and 21.09 (major) ppm. HRMS (pos.): calcd. for $\mathrm{C}_{18} \mathrm{H}_{31} \mathrm{~N}_{3} \mathrm{O}_{8} \mathrm{P}[\mathrm{M}+\mathrm{H}]^{+} 448.1843$; found 448.1855

\section{((6'-N-6'-Deoxy-(D)-galactopyranosyl))- $N$-(diethylphosphono-} methyl)-thymine-1-yl acetamide (46): 6'- $N$-6'-Deoxy-1,2;3,4-di$O$-isopropylidene- $(D)$-galactopyranosyl- $N$-(diethylphosphonomethyl)-thymine-1-yl acetamide $43(1.76 \mathrm{~g}, 3.06 \mathrm{mmol})$ was mixed with $80 \%$ aq. TFA $(50 \mathrm{ml})$. After $1 \mathrm{~h}$ TLC showed consumption of all the starting material. After removal of all the volatiles in vacuo and drying, water was added to the residual oil, followed by Amberlite IRA $400 \mathrm{OH}^{-}$until neutrality. The resin was filtered and washed with water. The combined filtrates were evaporated and dried in vacuo to leave a crude residue which was then purified by column chromatography $\left(\mathrm{CH}_{2} \mathrm{Cl}_{2}: \mathrm{MeOH} 80: 20,1 \% \mathrm{EtN}_{3}\right)$ to give compound 46 as a white foam $(1.31 \mathrm{~g}, 86 \%)$. The NMR spectra of 46 is very complex due to the couplings to ${ }^{31} \mathrm{P}$, the presence of syn/anti isomers around the amide bond and $\alpha / \beta$ anomers at $\mathrm{C}_{1},{ }^{1} \mathrm{H}$ NMR (500 MHz, MeOD): $\delta=7.34$ (major), 7.32 (minor), 7.30 (major) and 7.27 (minor) $(4 \mathrm{x} \mathrm{d}, J=1.2 \mathrm{~Hz}, 1 \mathrm{H}), 5.50$ (major, d, $J$ $=3.7 \mathrm{~Hz}$ ) and 5.17 (minor, $\mathrm{d}, J=2.4 \mathrm{~Hz}$ ) and 5.14 (minor, $\mathrm{d}, J=$ $3.2 \mathrm{~Hz})(1 \mathrm{H}), 4.92-4.77$ (unresolved m), $4.46(\mathrm{~d}, J=7.3 \mathrm{~Hz}, 0.5 \mathrm{H})$, 4.40-4.38 (minor) and 4.34-4.32 (major) $(2 \times \mathrm{m}, 1 \mathrm{H}), 4.25-4.00$ (unresolved $\mathrm{m}, 6.5 \mathrm{H}$ ), 3.94-3.92 (unresolved $\mathrm{m}, 1 \mathrm{H}$ ), 3.85-3.74 (unresolved $\mathrm{m}, 3 \mathrm{H}$ ), 3.64-3.58 (unresolved $\mathrm{m}, 1 \mathrm{H}$ ), 3.54-3.43 (unresolved m, 1.5 H), 3.31-3.29 (m, 0.5 H), $3.20(\mathrm{q}, J=7.4 \mathrm{~Hz}, 2$ $\mathrm{H}), 1.86$ (minor) and 1.85 (major) $(2 \mathrm{x} \mathrm{d}, J=1.1 \mathrm{~Hz}, 3 \mathrm{H}), 1.37$ (minor) and 1.30 (major) $(2 \mathrm{x} \mathrm{t}, J=7.2 \mathrm{~Hz}, 6 \mathrm{H}) \mathrm{ppm} .{ }^{13} \mathrm{C}$ NMR $(125 \mathrm{MHz}, \mathrm{MeOD}): \delta=170.0,169.8,167.0,166.9,153.1,153.0$, 
$143.8,143.7,110.9,110.8,98.8,98.7,94.2,94.1,74.8,74.7,74.1$, $73.6,72.8,71.5,71.3,71.0,70.9,70.8,70.6,70.2,69.3,68.4,64.5$, $64.23,64.17,64.1,64.0,53.6,49.9,49.8,49.7,47.8,42.9,42.3$ (major, d, $J_{\mathrm{CP}}=157.1 \mathrm{~Hz}$ ), 42.3 (major, d, $J_{\mathrm{CP}}=157.1 \mathrm{~Hz}$ ) and 42.2 (minor, d, $J_{\mathrm{CP}}=156.8 \mathrm{~Hz}$ ), 16.83, 16.79, 16.75. 16.70, 16.6, 12.2, 12.1. ${ }^{31} \mathrm{P}$ NMR (121 MHz, MeOD): $\delta=22.9,22.8,22.6$ (major) and 21.8 ppm. HRMS (pos.): calcd. for $\mathrm{C}_{18} \mathrm{H}_{30} \mathrm{~N}_{3} \mathrm{NaO}_{11} \mathrm{P}$ $[\mathrm{M}+\mathrm{Na}]^{+}$518.1510; found 518.1516.

$N$-(Diethylphosphonomethyl)- $N$-[(R)-2,3-dihydroxypropyl)]thymine-1-yl acetamide (47): To a stirred solution of (6'-N-6'deoxy-(D)-galactopyranosyl)- $N$-(diethylphosphonomethyl)thymine-1-yl acetamide $46(0.30 \mathrm{~g}, 0.61 \mathrm{mmol})$ in absolute ethanol $(8 \mathrm{ml})$ at $0{ }^{\circ} \mathrm{C}$ was added sodium periodate $(0.52 \mathrm{~g}, 2.43 \mathrm{mmol})$ solubilized in a small volume of water $(2.5 \mathrm{ml})$. The mixture turned immediately opaque. The reaction was completed after stirring for $1 \mathrm{~h}$, it was then filtered through a pad of Celite washing with ethanol and the filtrate was concentrated in vacuo at $25{ }^{\circ} \mathrm{C}$. The resulting white solid residue was then dried under reduced pressure and used in the following step without further purification. The crude residue was suspended in absolute ethanol and the mixture was cooled to $0{ }^{\circ} \mathrm{C}$. Sodium borohydride $(0.045 \mathrm{~g}, 1.19 \mathrm{mmol})$ was then added and the reaction mixture was allowed to warm to r.t. The stirring was continued for $3 \mathrm{~h}$. The reaction mixture was then quenched with $10 \% \mathrm{HOAc}$ until $\mathrm{pH}=7$. All the volatiles were removed in vacuo to leave a crude product which was purified by column chromatography $\left(\mathrm{CH}_{2} \mathrm{Cl}_{2}: \mathrm{MeOH} 9: 1\right)$ to give compound 47 $(0.11 \mathrm{~g}, 52 \%)$ as a white solid. A degradation fragment 49 was also isolated $(0.016 \mathrm{~g}, 8 \%)$, while traces $(<1 \%)$ of fragment 48 were identified by mass spectroscopy [HRMS (neg.): calcd. for $\mathrm{C}_{14} \mathrm{H}_{23} \mathrm{~N}_{3} \mathrm{O}_{7} \mathrm{P}$ [M-H] $]^{-}$376.1279; found 376.1266] but the compound was not fully characterized. Data for 47: ${ }^{1} \mathrm{H}$ NMR (500 $\mathrm{MHz}, \mathrm{D}_{2} \mathrm{O}$ ): $\delta=7.38$ (major) and 7.36 (minor) $(2 \mathrm{x} \mathrm{d}$, rotamers, $J=$ $1.1 \mathrm{~Hz}, 1 \mathrm{H}), 4.88$ (ddd, $J=1.7,17.0,35.6 \mathrm{~Hz}, 2 \mathrm{H}), 4.30-4.14$ (unresolved $\mathrm{m}, 5 \mathrm{H}), 4.09-4.07$ (major) and 4.03-3.98 (minor) $(2 \mathrm{x}$ $\mathrm{m}$, rotamers, $1 \mathrm{H}), 3.91(\mathrm{dd}, J=11.1,16.0 \mathrm{~Hz}, 1 \mathrm{H}), 3.70-3.51$ (unresolved $\mathrm{m}, 4 \mathrm{H}), 1.90(\mathrm{~s}, 3 \mathrm{H}), 1.38$ (minor, $\mathrm{t}, J=7.1 \mathrm{~Hz})$ and 1.33 (major, dt, $J=1.5,7.5 \mathrm{~Hz}$ ) (rotamers, $6 \mathrm{H}$ ) ppm. ${ }^{13} \mathrm{C}$ NMR $(75$ $\mathrm{MHz}, \mathrm{D}_{2} \mathrm{O}$ ): $\delta=169.0$ (minor) and 168.8 (major), 166.7, 151.9 (minor) and 151.8 (major), 142.8 (major) and 142.7 (minor), 110.6 (minor) and 110.5 (major), 69.1 (minor) and 68.5 (major), 64.3 (minor, d, $J_{\mathrm{CP}}=7.2 \mathrm{~Hz}$ ) and 63.9 (major, d, $J_{\mathrm{CP}}=6.7 \mathrm{~Hz}$ ), 62.9 (minor) and 62.6 (major), 50.1 (major) and 50.0 (minor), 49.2 (minor) and 49.1 (major), 43.6 (minor, $\mathrm{d}, J_{\mathrm{CP}}=156.9 \mathrm{~Hz}$ ) and 41.2 (major, d, $J_{\mathrm{CP}}=155.7 \mathrm{~Hz}$ ), 15.5 (minor, $\mathrm{d}, J_{\mathrm{CP}}=5.4 \mathrm{~Hz}$ ) and 15.3 (major, d, $\left.J_{\mathrm{CP}}=5.7 \mathrm{~Hz}\right), 11.0 \mathrm{ppm} .{ }^{31} \mathrm{P}$ NMR $\left(121 \mathrm{MHz}, \mathrm{D}_{2} \mathrm{O}\right): \delta=$ 24.0 (major) and 22.4 (minor) ppm. HRMS (pos.): calcd. for $\mathrm{C}_{15} \mathrm{H}_{26} \mathrm{~N}_{3} \mathrm{NaO}_{8} \mathrm{P}[\mathrm{M}+\mathrm{Na}]^{+} 430.1349$; found 430.1339. Data for 49: ${ }^{1} \mathrm{H}$ NMR (MeOD, $300 \mathrm{MHz}$ ): $\delta=7.35$ (d, $\left.J=1.2 \mathrm{~Hz}, 1 \mathrm{H}\right), 4.42$ (d, $J=1.4 \mathrm{~Hz}, 2 \mathrm{H}), 4.18-4.08(\mathrm{~m}, 4 \mathrm{H}), 3.72(\mathrm{~d}, J=11.7 \mathrm{~Hz}, 2 \mathrm{H})$, $3.20(\mathrm{q}, J=7.3 \mathrm{~Hz}, 1 \mathrm{H}), 1.86(\mathrm{~d}, J=1.1 \mathrm{~Hz}, 3 \mathrm{H}), 1.33-1.27(\mathrm{~m}, 6$ H) ppm. ${ }^{13} \mathrm{C}$ NMR (MeOD, $75 \mathrm{MHz}$,): $\delta=169.8,165.2,151.2$, $141.8,109.3,62.5\left(\mathrm{~d}, J_{\mathrm{CP}}=6.6 \mathrm{~Hz}\right), 49.0,33.9\left(\mathrm{~d}, J_{\mathrm{CP}}=158.3 \mathrm{~Hz}\right)$, $14.9\left(\mathrm{~d}, J_{\mathrm{CP}}=5.9 \mathrm{~Hz}\right), 10.5 \mathrm{ppm} .{ }^{31} \mathrm{P} \mathrm{NMR}(\mathrm{MeOD}, 121 \mathrm{MHz}): \delta=$ 22.8 ppm. HRMS (pos.): calcd. for $\mathrm{C}_{12} \mathrm{H}_{21} \mathrm{~N}_{3} \mathrm{O}_{6} \mathrm{P} \quad[\mathrm{M}+\mathrm{H}]^{+}$ 334.1163; found 334.1163.

$N$-[(R)-2,3-Dihydroxypropyl)]- $N$-(phosphonomethyl)-thymin-1yl acetamide (50): To a solution of $N$-(diethylphosphonomethyl)$N$-[(R)-2,3-dihydroxypropyl)]-thymine-1-yl acetamide $47(0.11 \mathrm{~g}$, $0.27 \mathrm{mmol})$ in dry $\mathrm{CH}_{2} \mathrm{Cl}_{2}(5 \mathrm{ml})$ at $0{ }^{\circ} \mathrm{C}$ was added iodotrimethylsilane $(0.15 \mathrm{ml}, 1.08 \mathrm{mmol})$. The reaction mixture was stirred overnight at r.t. under an argon atmosphere. It was then quenched with a $1 \mathrm{M}$ TEAB solution and evaporated under reduced pressure. The crude residue was first purified by column chromatography $\left(\mathrm{CH}_{2} \mathrm{Cl}_{2}: \mathrm{MeOH}: 1 \mathrm{M}\right.$ TEAB $\left.5: 2.5: 0.5\right)$ and then by
HPLC using a Source 15Q anion exchange column (gradient $1 \mathrm{M}$ TEAB from 1 to $25 \%$ in $30 \mathrm{~min}$ ) to afford the title compound $\mathbf{5 0}$ as a triethylammonium salt $(76 \%) . \alpha_{D}+19.1^{\circ}$, c 0.52 , DMSO-PBS; ${ }^{1} \mathrm{H}$ NMR (300 MHz, $\mathrm{D}_{2} \mathrm{O}$ ): $\delta=7.41$ (major, $\mathrm{d}, J=1.1 \mathrm{~Hz}$ ) and 7.35 (minor, br s) (rotamers, $1 \mathrm{H}, \mathrm{H}_{6}$ ), 4.91 (br s, $2 \mathrm{H}, \mathrm{COCH}_{2}$ ), 4.164.08 (minor) and 4.02-3.95 (major) $(2 \mathrm{x} \mathrm{m}$, rotamers, $1 \mathrm{H}), 3.74$ (minor) and 3.69 (major) $(2 \mathrm{x} \mathrm{d}$, rotamers, $J=4.2 \mathrm{~Hz}, 1 \mathrm{H}), 3.67$ 3.47 (unresolved m, $5 \mathrm{H}), 3.14$ (q, $J=7.3 \mathrm{~Hz}, 12 \mathrm{H},{ }^{+} \mathrm{N}\left(\mathrm{CH}_{2} \mathrm{CH}_{3}\right)_{3}$ ), $1.86\left(\mathrm{~s}, 3 \mathrm{H}, \mathrm{CH}_{3}\right), 1.25$ (t, $\left.J=7.3 \mathrm{~Hz}, 18 \mathrm{H},{ }^{+} \mathrm{N}\left(\mathrm{CH}_{2} \mathrm{CH}_{3}\right)_{3}\right) \mathrm{ppm}$. ${ }^{13} \mathrm{C}$ NMR (75 MHz, $\left.\mathrm{D}_{2} \mathrm{O}\right): \delta=169.6,168.9,154.3,143.0,110.4$, 69.3 (major) and 68.4 (minor), 63.1 (major) and 63.0 (minor), 50.7, 49.5, $47.9\left(\mathrm{~d}, J_{\mathrm{CP}}=136.9 \mathrm{~Hz}\right), 46.3,11.3,8.00 \mathrm{ppm} .{ }^{31} \mathrm{P}$ NMR $(121$ $\mathrm{MHz}, \mathrm{D}_{2} \mathrm{O}$ ): $\delta=12.6$ (minor) and 12.0 (major) ppm. HRMS (neg.): calcd. for $\mathrm{C}_{11} \mathrm{H}_{17} \mathrm{~N}_{3} \mathrm{O}_{8} \mathrm{P}$ [M-H] 350.0759 ; found 350.0765 .

\section{$N$-[(S)-2,3-Dihydroxypropyl)]- $N$-(phosphonomethyl)-thymin-1-} yl acetamide (51): To a solution of (S)- $N$ (diethylphosphonomethyl)-3- $N$-(1,2-O-isopropylidenepropyl)thymine-1-yl acetamide $42(0.10 \mathrm{~g}, 0.22 \mathrm{mmol})$ in dry $\mathrm{CH}_{2} \mathrm{Cl}_{2}(5$ $\mathrm{ml})$ at $0{ }^{\circ} \mathrm{C}$ was added triethylamine $(0.31 \mathrm{ml}, 2.23 \mathrm{mmol})$, followed by iodotrimethylsilane $(0.25 \mathrm{ml}, 1.80 \mathrm{mmol})$. The reaction mixture was stirred overnight under an argon atmosphere. It was then quenched with a $1 \mathrm{M}$ TEAB solution and evaporated under reduced pressure. The crude residue was first purified by column chromatography $\left(\mathrm{CH}_{2} \mathrm{Cl}_{2}: \mathrm{MeOH}: 1 \mathrm{M}\right.$ TEAB 5:2.5:0.5) and then by HPLC using a Source $15 \mathrm{Q}$ anion exchange column (gradient $1 \mathrm{M}$ TEAB from 1 to $25 \%$ in $30 \mathrm{~min}$ ) to afford the title compound 51 as triethylammonium salt $(78 \%)$. $\alpha_{D}-9.8^{\circ}$, c 0.88 , DMSO-PBS; ${ }^{1} \mathrm{H}$ NMR (300 MHz, $\mathrm{D}_{2} \mathrm{O}$ ): $\delta=7.41$ (major) and 7.35 (minor) $(2 \mathrm{x}$ br s, rotamers, $1 \mathrm{H}), 4.91(\mathrm{br} \mathrm{s}, 2 \mathrm{H}), 3.98-3.96(\mathrm{~m}, 1$ $\mathrm{H}), 3.74$ (minor) and 3.69 (major) $(2 \mathrm{x} \mathrm{d}$, rotamers, $J=4.3 \mathrm{~Hz}, 1$ $\mathrm{H}), 3.66-3.48(\mathrm{~m}$, unresolved, $5 \mathrm{H}), 3.14(\mathrm{q}, J=7.4 \mathrm{~Hz}, 12 \mathrm{H}), 1.86$ $(\mathrm{s}, 3 \mathrm{H}), 1.24(\mathrm{t}, J=7.4 \mathrm{~Hz}, 18 \mathrm{H}) \mathrm{ppm} .{ }^{13} \mathrm{C}$ NMR $\left(75 \mathrm{MHz}, \mathrm{D}_{2} \mathrm{O}\right)$ : $\delta=169.5,169.2,154.2,143.4,110.7,69.6$ (major) and 68.7 (minor), 63.4 (major) and 63.3 (minor), 51.0, 49.8, 48.1 (d, $\left.J_{\mathrm{CP}}=136.9 \mathrm{~Hz}\right)$, 46.6, 11.6, 8.31 ppm. ${ }^{31} \mathrm{P}$ NMR (121 MHz, $\mathrm{D}_{2} \mathrm{O}$ ): $\delta=12.6$ (minor) and 12.0 (major) ppm. HRMS (neg.): calcd. for $\mathrm{C}_{11} \mathrm{H}_{17} \mathrm{~N}_{3} \mathrm{O}{ }_{8} \mathrm{P}$ [M$\mathrm{H}]^{-} 350.0759$; found 350.0760 .

$N$-[(Rac)-2,3-dihydroxypropyl)]- $N$-(phosphonomethyl)-thymin1-yl acetamide (52): To a solution of (rac) $-N$ (diethylphosphonomethyl)-3- $N$-(1,2-O-isopropylidenepropyl)-

thymine-1-yl acetamide $45(0.40 \mathrm{~g}, 0.89 \mathrm{mmol})$ in dry $\mathrm{CH}_{2} \mathrm{Cl}_{2}(10$ $\mathrm{ml})$ at $0{ }^{\circ} \mathrm{C}$ was added triethylamine $(1.24 \mathrm{ml}, 8.89 \mathrm{mmol})$, followed by iodotrimethylsilane $(0.76 \mathrm{ml}, 5.35 \mathrm{mmol})$. The reaction mixture was stirred overnight under an argon atmosphere. It was then quenched with a $1 \mathrm{M}$ TEAB solution and evaporated under reduced pressure. The crude residue was first purified by column chromatography $\left(\mathrm{CH}_{2} \mathrm{Cl}_{2}: \mathrm{MeOH}: 1 \mathrm{M}\right.$ TEAB 5:2.5:0.5) and then by HPLC using a Source $15 \mathrm{Q}$ anion exchange column (gradient $1 \mathrm{M}$ TEAB from 1 to $25 \%$ in $30 \mathrm{~min}$ ) to afford the title compound 52 as triethylammonium salt $(90 \%)$. ${ }^{1} \mathrm{H}$ NMR (300 $\mathrm{MHz}, \mathrm{D}_{2} \mathrm{O}$ ): $\delta=7.33$ (major, d, $J=1.1 \mathrm{~Hz}$ ) and 7.27 (minor, br s) (rotamers, 1H), 4.83 (br s, 2H), 4.08-3.99 (minor) and 3.92-3.86 (major) $(2 \mathrm{x} \mathrm{m}, 1 \mathrm{H}), 3.66$ (minor) and 3.61 (major) $(2 \mathrm{x} \mathrm{d}$, rotamers, $J=4.3 \mathrm{~Hz}, 1 \mathrm{H}), 3.54-3.38$ (m, unresolved, $5 \mathrm{H}), 3.01$ (q, $J=7.3 \mathrm{~Hz}$, $12 \mathrm{H}), 1.78(\mathrm{~s}, 3 \mathrm{H}), 1.16(\mathrm{t}, J=7.3 \mathrm{~Hz}, 18 \mathrm{H}) \mathrm{ppm} .{ }^{13} \mathrm{C}$ NMR $(75$ $\mathrm{MHz}, \mathrm{D}_{2} \mathrm{O}$ ): $\delta=169.5,168.9,154.2,143.0,110.4,69.2$ (major) and 68.4 (minor), 63.1 (major) and 62.9 (minor), 50.6, 49.5, 47.8 (d, $\left.J_{\mathrm{CP}}=136.8 \mathrm{~Hz}\right), 46.2,11.3,7.95 \mathrm{ppm} .{ }^{31} \mathrm{P}$ NMR $\left(121 \mathrm{MHz}, \mathrm{D}_{2} \mathrm{O}\right)$ : $\delta=12.6$ (minor) and 12.0 (major) ppm. HRMS (neg.): calcd. for $\mathrm{C}_{11} \mathrm{H}_{17} \mathrm{~N}_{3} \mathrm{O}_{8} \mathrm{P}[\mathrm{M}-\mathrm{H}]^{-} 350.0759$; found 350.0762 .

$N$-(Diethylphosphonomethyl)- $N$-(1,3-dihydroxypropyl)-thymin1-yl acetamide (53): To a solution of 2- $\mathrm{N}$ (diethylphosphonomethyl)-(1,3-bis-tbutyldiphenylsilyloxypropyl)- 
thymine-1-yl acetamide $44(0.20 \mathrm{~g}, 0.23 \mathrm{mmol})$ in dry DMF (2 ml) at $0{ }^{\circ} \mathrm{C}$ was added a solution of tris(dimethylamino)sulfonium difluorotrimethylsilicate (TAS-F, $0.15 \mathrm{~g}, 0.54 \mathrm{mmol}$ ) in dry DMF $(2 \mathrm{ml})$. The reaction mixture was stirred at $0{ }^{\circ} \mathrm{C}$ for $2 \mathrm{~h}$ and then warmed to r.t.. It was then diluted with EtOAc and washed with $\mathrm{pH}$ 7 buffer. The aqueous layer was extracted with EtOAc and the combined organic layers were dried over $\mathrm{Na}_{2} \mathrm{SO}_{4}$, filtered and concentrated in vacuo. The resulting crude residue was purified by column chromatography $\left(\mathrm{CH}_{2} \mathrm{Cl}_{2}: \mathrm{MeOH} 9: 1\right)$ to give the title compound $53(0.050 \mathrm{~g}, 54 \%)$ as a white solid. ${ }^{1} \mathrm{H}$ NMR (300 MHz, MeOD): $\delta=7.27$ (major, d, $J=1.2 \mathrm{~Hz}$ ) and 7.25 (minor, br s) (rotamers, $1 \mathrm{H}$ ), 4.80 (apparent d, $J=1.6 \mathrm{~Hz}, 2 \mathrm{H}$ ), 4.26-4.18 (minor) and 4.17-4.08 (major) (2 x m, rotamers, $4 \mathrm{H}), 4.03-3.97$ (m, $1 \mathrm{H}), 3.87$ (d, $J=12.0 \mathrm{~Hz}, 2 \mathrm{H}), 3.70-3.68(\mathrm{~m}, 2 \mathrm{H}), 3.33-3.28$ (m, $2 \mathrm{H}), 1.86$ (major) and 1.85 (minor) $(2 \mathrm{x} \mathrm{s}$, rotamers, $3 \mathrm{H}), 1.38$ and $1.30(2 \mathrm{x} \mathrm{t}$, rotamers, $J=7.1 \mathrm{~Hz}, 6 \mathrm{H}) \mathrm{ppm} .{ }^{13} \mathrm{C} \mathrm{NMR}(75 \mathrm{MHz}$, MeOD): $\delta=169.0,165.3,151.3,142.0,109.2,62.5\left(\mathrm{~d}, J_{\mathrm{CP}}=6.6\right.$ $\mathrm{Hz}), 61.1,59.5$ (minor) and 58.6 (major), 48.4, $37.7\left(\mathrm{~d}, J_{\mathrm{CP}}=158.9\right.$ $\mathrm{Hz})$, 14. $9\left(\mathrm{~d}, J_{\mathrm{CP}}=6.2 \mathrm{~Hz}\right), 10.5 \mathrm{ppm} .{ }^{31} \mathrm{P} \mathrm{NMR}(121 \mathrm{MHz}$, $\mathrm{MeOD}$ ): $\delta=25.6$ (major) and 22.0 (minor) ppm. HRMS (pos.): calcd. for $\mathrm{C}_{15} \mathrm{H}_{26} \mathrm{~N}_{3} \mathrm{NaO}_{8} \mathrm{P}[\mathrm{M}+\mathrm{Na}]^{+} 430.1350$; found 430.1350 .

\section{$N$-(1,3-Dihydroxypropyl)- $N$-(phosphonomethyl)-thymin-1-yl}

acetamide (54): To a solution of $N$-(diethylphosphonomethyl)- $N$ (1,3-dihydroxypropyl)-thymin-1-yl acetamide 53 (0.05 g, 0.12 mmol $)$ in dry $\mathrm{CH}_{2} \mathrm{Cl}_{2}(3 \mathrm{ml})$ at $0{ }^{\circ} \mathrm{C}$ was added triethylamine $(0.17$ $\mathrm{ml}, 1.18 \mathrm{mmol})$, followed by iodotrimethylsilane $(0.07 \mathrm{ml}, 0.49$ $\mathrm{mmol})$. The reaction mixture was stirred overnight at r.t. under an argon atmosphere. It was then quenched with a $1 \mathrm{M}$ TEAB solution and evaporated under reduced pressure. The crude residue was first purified by column chromatography $\left(\mathrm{CH}_{2} \mathrm{Cl}_{2}: \mathrm{MeOH}: 1 \mathrm{M}\right.$ TEAB $5: 2.5: 0.5)$ and then by HPLC using a Source $15 \mathrm{Q}$ anion exchange column (gradient $1 \mathrm{M}$ TEAB from 1 to $25 \%$ in $30 \mathrm{~min}$ ) to afford the title compound 54 as a triethylammonium salt $(82 \%) .{ }^{1} \mathrm{H}$ NMR (300 MHz, $\mathrm{D}_{2} \mathrm{O}$ ): $\delta=7.49$ (major, d, $J=1.1 \mathrm{~Hz}$ ) and 7.42 (minor, br s) $\left(1 \mathrm{H}\right.$, rotamers, $\left.\mathrm{H}_{6}\right), 5.06\left(\mathrm{br} \mathrm{s}, 2 \mathrm{H}, \mathrm{COCH}_{2}\right), 4.45-4.40(\mathrm{~m}, 1$ $\mathrm{H}, \mathrm{CH}), 3.81\left(\mathrm{dd}, J=12.3,8.6 \mathrm{~Hz}, 2 \mathrm{H}, \mathrm{CH}_{2}\right), 3.67(\mathrm{dd}, J=12.3$, $\left.4.7 \mathrm{~Hz}, 2 \mathrm{H}, \mathrm{CH}_{2}\right), 3.46$ (d, $\left.J=10.3 \mathrm{~Hz}, 2 \mathrm{H}, \mathrm{POCH}_{2}\right), 3.20$ (q, $J=$ $\left.7.3 \mathrm{~Hz}, 12 \mathrm{H},{ }^{+} \mathrm{N}\left(\mathrm{CH}_{2} \mathrm{CH}_{3}\right)_{3}\right), 1.89$ (minor) and 1.88 (major) (s, $3 \mathrm{H}$, $\left.\mathrm{CH}_{3}\right), 1.27\left(\mathrm{t}, J=7.3 \mathrm{~Hz}, 18 \mathrm{H},{ }^{+} \mathrm{N}\left(\mathrm{CH}_{2} \mathrm{CH}_{3}\right)_{3}\right) \mathrm{ppm} .{ }^{13} \mathrm{C} \mathrm{NMR}(75$ $\left.\mathrm{MHz}, \mathrm{D}_{2} \mathrm{O}\right): \delta=169.8,167.1,152.3,143.3,110.4,61.4,58.6$ (major) and 57.8 (minor), 49.9, 46.3, $44.7\left(\mathrm{~d}, J_{\mathrm{CP}}=134.9 \mathrm{~Hz}\right), 11.0$, 7.92 ppm. ${ }^{31} \mathrm{P}$ NMR (121 MHz, $\mathrm{D}_{2} \mathrm{O}$ ): $\delta=13.7$ (minor) and 12.3 (major) ppm. HRMS (neg.): calcd. for $\mathrm{C}_{11} \mathrm{H}_{17} \mathrm{~N}_{3} \mathrm{O}_{8} \mathrm{P}[\mathrm{M}-\mathrm{H}]^{-}$, 350.0759 ; found 350.0766 .

[1] J. C. Chaput, H. Yu, S. Zhang, Chem. Biol. 2012, 19, 1360-1371.

[2] K. U. Schoning, P. Scholz, S. Guntha, X. Wu, R. Krishnamurthy, A. Eschenmoser, Science 2000, 290, 1347-1351.

[3] P. E. Nielsen, Acc. Chem. Res. 1999, 32, 624-630.

[4] E. Meggers, L. Zhang, Acc. Chem. Res. 2010, 43, 1092-1102.

[5] P. E. Nielsen, Chem. Biodivers. 2010, 7, 786-804.

[6] P. E. Nielsen, M. Egholm, R. H. Berg, O. Buchardt, Science 1991, 254, 1497-1500.

[7] M. Egholm, O. Buchardt, L. Christensen, C. Behrens, S. M. Freier, D. A. Driver, R. H. Berg, S. K. Kim, B. Norden, P. E. Nielsen, Nature 1993, 365, 566-568.

[8] A. Peyman, E. Uhlmann, K. Wagner, S. Augustin, G. Breipohl, D. W. Will, A. Schafer, H. Wallmeier, Angew. Chem. Int. Ed. Engl. 1996, $35,2636-2638$.
[9] A. C. vanderLaan, R. Stromberg, J. H. vanBoom, E. KuylYeheskiely, V. A. Efimov, O. G. Chakhmakhcheva, Tetrahedron Lett. 1996, 37, 7857-7860

[10] V. A. Efimov, M. V. Choob, A. L. Kalinkina, O. G. Chakhmakhcheva, R. Stromberg, A. C. VanDerLaan, N. J. Meeuwenoord, E. KuylYeheskiely, J. H. VanBoom, Collect. Czech. Chem. Commun. 1996, 61, S262-S264.

[11] V. A. Efimov, M. V. Choob, A. A. Buryakova, O. G. Chakhmakhcheva, Nucleosides Nucleotides 1998, 17, 1671-1679.

[12] J. Kehler, U. Henriksen, H. Vejbjerg, O. Dahl, Bioorg. Med. Chem. 1998, 6, 315-322.

[13] A. Peyman, E. Uhlmann, K. Wagner, S. Augustin, C. Weiser, D. W. Will, G. Breipohl, Angew. Chem. Int. Ed. Engl. 1997, 36, 2809-2812.

[14] V. A. Efimov, M. V. Choob, A. A. Buryakova, A. L. Kalinkina, O. G. Chakhmakhcheva, Nucleic Acids Res. 1998, 26, 566-575.

[15] V. A. Efimov, O. G. Chakhmakhcheva, E. Wickstrom, Nucleos Nucleot Nucl 2005, 24, 1853-1874.

[16] M. Renders, G. Emmerechts, J. Rozenski, M. Krecmerova, A. Holy, P. Herdewijn, Angew. Chem. 2007, 46, 2501-2504.

[17] A. V. Azhayev, M. L. Antopolsky, Tetrahedron 2001, 57, 4977-4986.

[18] H. Ouyang, S. L. Morris-Natschke, K. S. Ishaq, P. Ward, D. Liu, S. Leonard, D. R. Thakker, J. Med. Chem. 2002, 45, 2857-2866.

[19] B. Doboszewski, P. Herdewijn, Tetrahedron Lett. 2011, 52, 38533855 .

[20] S. David, S. Hanessian, Tetrahedron 1985, 41, 643-663.

[21] J. P. Kutney, A. H. Ratcliffe, Synth. Commun. 1975, 5, 47-52.

[22] K. C. K. Swamy, N. N. B. Kumar, E. Balaraman, K. V. P. P. Kumar, Chem. Rev. 2009, 109, 2551-2651.

[23] J. M. Macdonald, H. T. Horsley, J. H. Ryan, S. Saubern, A. B. Holmes, Org. Lett. 2008, 10, 4227-4229.

[24] D. Terakado, H. Koutaka, T. Oriyama, Tetrahedron: Asymmetry 2005, 16, 1157-1165.

[25] Y. Gao, R. M. Hanson, J. M. Klunder, S. Y. Ko, H. Masamune, K. B. Sharpless, J. Am. Chem. Soc. 1987, 109, 5765-5780.

[26] M. Pallavicini, E. Valoti, L. Villa, O. Piccolo, Tetrahedron: Asymmetry 1994, 5, 5-8.

[27] W. L. Nelson, J. E. Wennerstrom, S. R. Sankar, J. Org. Chem. 1977, 42, 1006-1012.

[28] E. A. Losey, M. D. Smith, M. Meng, M. D. Best, Bioconj. Chem. 2009, 20, 376-383.

[29] T. Sato, T. Mizutani, Y. Okumura, T. Fujisawa, Tetrahedron Lett. 1989, 30, 3701-3702.

[30] S. Nagarajan, B. Ganem, J. Org. Chem. 1987, 52, 5044-5046.

[31] F. S. Gibson, M. S. Park, H. Rapoport, J. Org. Chem. 1994, 59, 7503-7507.

[32] K. Danielmeier, E. Steckhan, Tetrahedron: Asymmetry 1995, 6, 1181-1190.

[33] G. J. Wang, R. I. Hollingsworth, J. Org. Chem. 1999, 64, 1036-1038.

[34] C. E. Ballou, H. O. L. Fischer, J. Am. Chem. Soc. 1954, 76, 31883193.

[35] R. S. Tipson, Methods Carbohydr. Chem. 1963, 2, 246-250.

[36] J. A. May, A. C. Sartorelli, J. Med. Chem. 1979, 22, 971-976.

[37] W. A. Szarek, G. W. Hay, B. Doboszewski, J. Chem. Soc., Chem. Commun. 1985, 663-664.

[38] B. Doboszewski, G. W. Hay, W. A. Szarek, Can. J. Chem. 1987, 65, 412-419.

[39] B. Doboszewski, P. R. da Silva, A. Y. Nazarenko, V. N. Nemykin, Acta Cryst. E 2010, 66, 3217-3218.

[40] B. Streicher, B. Wunsch, Carbohydr. Res. 2003, 338, 2375-2385. 
[41] J. J. Bronson, L. M. Ferrara, H. G. Howell, P. R. Brodfuehrer, J. C. Martin, Nucleosides Nucleotides 1990, 9, 745-769.

[42] X. Creary, T. L. Underiner, J. Org. Chem. 1985, 50, 2165-2170.

[43] J. M. Vatele, S. Hanessian, Tetrahedron 1996, 52, 10557-10568.

[44] H. A. Staab, K. Wendel, Liebigs Ann. Chem. 1966, 694, 86-90.

[45] A. S. Jones, P. Lewis, S. F. Withers, Tetrahedron 1973, 29, 2293 2296.

[46] K. A. Scheidt, H. Chen, B. C. Follows, S. R. Chemler, D. S. Coffey, W. R. Roush, J. Org. Chem. 1998, 63, 6436-6437.
[47] D. P. Phillion, S. S. Andrew, Tetrahedron Lett. 1986, 27, 1477-1480. 
The $\mathrm{C} 5$ atom in 6-amino-6-

deoxygalactopyranose was used as a source of chirality to obtain (*), whereas its enantiomer and achiral were conveniently synthesized starting from (S)-3-aminoglycerol and 2-aminoglycerol, respectively. The final compounds represent new monomeric precursors to backbone-modified nucleic acids of the pPNA-type.

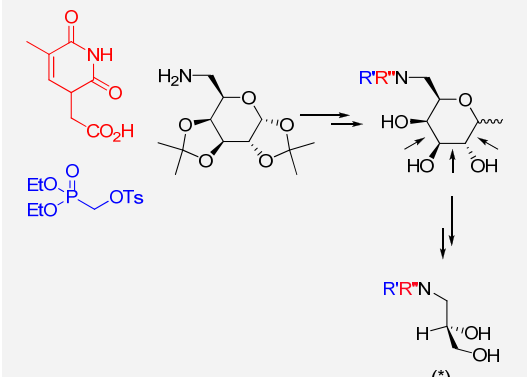

(*)
Bogdan Doboszewski, Elisabetta Groaz, and Piet Herdewijn. Page No. - Page No.

Synthesis of new phosphonoglycine backbone units for the development of unnatural phosphono peptide nucleic acids.

Keywords: Nucleotides/Phosphonates/ Phosphono peptide nucleic acid (pPNA) 